\title{
Asymptotic analysis, polarization matrices and topological derivatives for piezoelectric materials with small voids
}

\author{
G.Cardone \\ University of Sannio - Department of Engineering \\ Corso Garibaldi, 107 - 82100 Benevento, Italy \\ email: giuseppe.cardone@unisannio.it \\ S.A.Nazarov \\ Institute of Mechanical Engineering Problems \\ V.O., Bolshoi pr., 61, 199178, St. Petersburg, Russia. \\ email: srgnazarov@yahoo.co.uk \\ J. Sokolowski \\ Institut Elie Cartan, UMR 7502 Nancy-Université-CNRS-INRIA \\ Laboratoire de Mathématiques, Université Henri Poincaré \\ Nancy 1, B.P. 239, 54506 Vandoeuvre lés Nancy Cedex, France \\ email: Jan.Sokolowski@iecn.u-nancy.fr
}

September 7, 2018

\begin{abstract}
Asymptotic formulae for the mechanical and electric fields in a piezoelectric body with a small void are derived and justified. Such results are new and useful for applications in the field of design of smart materials. In this way the topological derivatives of shape functionals are obtained for piezoelectricity. The asymptotic formulae are given in terms of the so-called polarization tensors (matrices) which are determined by the integral characteristics of voids. The distinguished feature of the piezoelectricity boundary value problems under considerations is the absence of positive definiteness of an differential operator which is non self-adjoint. Two specific Gibbs' functionals of the problem are defined by the energy and the electric enthalpy. The topological derivatives are defined in different manners for each of the governing functionals. Actually, the topological derivative of the enthalpy functional is local i.e., defined by the pointwise values of the governing fields, in contrary to the energy functional and some other suitable shape functionals which admit non-local topological derivatives, i.e., depending on the whole problem data. An example with the weak interaction between mechanical and electric fields provides the explicit asymptotic expansions and can be directly used in numerical procedures of optimal design for smart materials.
\end{abstract}

Key words: Piezoelectricity, polarization matrix, asymptotic analysis, electric enthalpy, topological derivative, optimum design, shape optimization.

MSC (2000): Primary 35Q30, 49J20, 76N10; Secondary 49Q10, 74P15.

\section{Introduction}

The paper is devoted to the asymptotic analysis of boundary value problems for coupled models. The coupling occurs between the mechanical part which takes the form of the linearized elasticity and governs 
the stress-strain state of the body, and the electrical part which describes the electromagnetic field in the body.

From the view point of applications, piezoelectric materials are of common use in electromechanical sensors and actuators, e.g., ultrasound transducers in medical imaging and therapy, force and acceleration sensors, positioning sensors, surface acoustic wave filters, still with the growing range of applications in modern technology. Their mode of action is based on the piezoelectric effect, that couples the electrical and mechanical behavior of such materials. For the optimal design of piezoelectric devices, efficient numerical procedures for shape and topology optimization should be still developed. In the modern theory of shape optimization it is required that the derivation of shape and topological derivatives of shape functionals to be optimized is performed beforehand. From one side, the derivation of shape gradients of integral functionals in smooth domains [49] and non-smooth domains [28] (cf. [40, 41]) has become a standard procedure. There is no major difficulty to perform such a shape sensitivity analysis for the elliptic boundary value problem under considerations. However, the boundary value problem in piezoelectricity cannot be posed in such a way that it simultaneously is formally self-adjoint and possesses a semi-bounded quadratic form. This specific feature makes the problem more involved from the asymptotic analysis point of view compared to the pure elasticity or pure electricity boundary value problems. In addition, the general case of inhomogeneous and anisotropic body is considered, which also requires for additional and new technicalities in asymptotic procedures which is the main subject of the paper. In particular, different formulations of the piezoelectricity problem (cf. Sections 2.3 2.4, 3.3) lead to two definitions of the polarization matrices which differ one from another by its properties. Moreover, only the electrical enthalpy, which is but the governing functional for the piezoelectric media (see, e.g., [12, 15, 46]) admits the topological derivative dependent on local characteristics of mechanical and electrical fields. Other shape functionals, especially the energy functional, get the topological derivatives dependent on the global characteristics of mechanical and electrical fields. This acquired trait raises the natural question on the properties of material derivatives for piezoelectricity in the framework of the shape sensitivity analysis with smooth or non-smooth boundary variations, it is clear that the result could be of the same nature, since the topological derivatives can be identified from the first order shape gradients by a limit passage e.g. in elasticity, [51] (cf. also [14]).

In the paper, we restrict ourselves to the asymptotic procedures of singular domain perturbations which allow us to obtain, in a natural way, the topological derivatives of shape functionals. In principle, the method developed here can be generalized to characterize the influence on solutions of the nonsmooth boundary variations, therefore, we can derive the shape gradients even in such a case, e.g., for small defects located close-by the boundary, including micro-cracks (see [42]).

Without entering into details, but with the strong practical implications in mind, we can claim that some possible applications of shape optimization in the field concern the design of electro-acoustic transducers which are constructed with piezoelectric actuator-patches and capacitative micro-machined ultrasound transducers. The task for optimal design for a class of electrostatic-mechanical-acoustic transducers can be e.g., the topology of electro-acoustic material and the topology of the electrodelayers, in order to achieve a maximal acoustic pressure, or a maximal acoustic energy in a specific sub-domains of the hold-all-domain. We refer the reader e.g. to [46, 12, 15] for modeling of piezoelectric materials, to 18 for material tensor identification for such materials, and to [19] for control issues.

Our aim is a possible application in shape optimization, thus we introduce the so-called topological derivatives of shape functionals for piezoelectric materials. It seems that the models are not up to now used in applied mathematics for the purposes of shape optimization, although the smart materials are of common use in the engineering practice. In shape optimization, the modern approach to numerical solution, requires the preliminary knowledge of explicit formulae for shape gradients [49] as well as of the topological derivatives [50, 40, 10. These formulae are used in the level-set-type methods which model the geometrical domain evolution by a zero-level set of solutions to non-linear hyperbolic equations of the Hamilton-Jacoby type. The shape gradient are present as the coefficients of the equations, and the topological derivatives are used to improve the values of the shape functional under consideration by the appropriate topology changes, e.g., for the minimization of the shape functional, the minima of the topological derivative of the functional indicate the location of a new hole in the geometrical domain 


\section{Preliminaries. Problem formulation and description of re- sults}

\subsection{Shape optimization in piezoelectricity}

This paper is motivated by the fact that, among numerous publications on shape optimization, shape sensitivity analysis for piezoelectric bodies does not exist, although piezoelectric materials are of extremely wide usage in the modern technologies, one can think of a simple lighter, available in any supermarket, or an elaborated computer work-station in a university. One, and definitely not the only one, distinguishing feature of such smart materials implies an easy energy transfer in both directions from mechanical fields to electric fields. The mathematical modeling of such a phenomenon leads to serious complications of analysis for governing partial differential equations because the corresponding boundary value problem is not formally self-adjoint in contrast to the boundary value problems for purely elastic bodies or purely electromagnetic media. This fact requires for the development of new mathematical tools and a careful choice of the cardinal shape functional while neglecting of non-self-adjointness provokes mistakes in both, mathematical formulae and physical interpretation of the obtained results (see Remark 21 below).

Introduced in [50] 1 , the topological derivative $T\left(u^{0} ; \omega_{1}\right)$ of a shape functional $\mathcal{J}$ is intended to describe the change of the functional $\mathcal{J}$ due to nucleation of holes or voids and allows to extend possible variations of the shape in an optimization process [2, 8, 9, in comparison with classical tools (cf. 49, 5, 6),

$$
\mathcal{J}\left(u^{h} ; \Omega(h)\right)=\mathcal{J}(u ; \Omega)+h^{\kappa} \mathcal{T}\left(u ; \omega_{1}\right)+o\left(h^{\kappa}\right), h \rightarrow+0,
$$

In (2.1), $h>0$ is a small parameter, i.e., the diameter of the opening $\omega_{h}$ in the entire body $\Omega \subset \mathbb{R}^{n}$, $u^{h}$ and $u$ are solutions of the boundary value problem in $\Omega(h)=\Omega \backslash \bar{\omega}_{h}$ and $\Omega$, respectively, and the exponent $\kappa>0$ depends on the space dimension $n$ and boundary conditions imposed on the interior $(n-1)$-dimensional surface $\partial \omega_{h}$.

Asymptotic analysis of elliptic problems in singularly perturbed domains, e.g., methods of matched and compound asymptotic expansions (cf. [16] and [28, respectively), has become the most appropriate and relevant to obtain almost explicit formulae for the topological derivatives as it has been demonstrated in 40, 41] and others. We also mention books [32, 3] where the subject is studied, to some extend, from physical and numerical point of view.

Strangely enough, only self-adjoint problems were heretofore examined carefully, although the fullblown approach in [28] can work for general boundary value problems for elliptic systems. In this paper we partly fill this gap by adapting formula (2.1) to certain shape functionals for a piezoelectric body.

The piezoelectricity problems admits two different formulations with non-symmetric and symmetric but non-semibounded quadratic forms, the energy and electric enthalpy functionals, respectively. By means of the Lax-Milgram lemma, the first formulation furnishes the existence and uniqueness result. At the same time, the topological derivative of the energy functional is a non-local characteristics of the piezoelectricity solutions in contrast to the pure elasticity problem (see Remark 21 below), while the absence of this intrinsic property is not caused by an incorrect definition (2.1) but occurs as well for the energy release rate in mechanics of cracks for piezoelectric media (see Remark 21 again). The fair explanation, we refer the reader to [52] for the modeling issues, of the latter refers to the electric enthalpy as one of Gibbs' functional obtained from the energy functional by the partial Lagrange transform on the electric components. This is the electric enthalpy $\mathcal{E}\left(u^{h} ; \Omega(h)\right.$ ) (see the definition in (2.20)), that governs the mechanical electric state of the piezoelectric body $\Omega(h)$ and, therefore, the second formulation becomes variational and provides the clear interpretation of the topological derivative $\mathcal{T}_{\mathcal{E}}\left(u ; \omega_{1}\right)$ in

$$
\mathcal{E}\left(u_{h} ; \Omega(h)\right)=\mathcal{E}(u ; \Omega)+h^{3} \mathcal{T}_{\mathcal{E}}\left(u ; \omega_{1}\right)+O\left(h^{4}\right), h \rightarrow+0 .
$$

\footnotetext{
${ }^{1}$ Actually, asymptotic formulae of type (2.1) together with the whole asymptotic series for energy functionals under various singular boundary perturbations had been derived much earlier in [27, although the notion of the topological derivative is due to [50.
} 
The indicated peculiarity of the piezoelectricity problem crucially influences topological derivatives of other shape functionals, too. For example, the traditional adjoint state (cf. [5, 49, 50]) has to be found out in the formally adjoint boundary value problem that occasionally underlines its name.

All the above observations lifts the piezoelectricity problem on the top of the list of unsolved problems in shape optimization, it seems that even the classical formulae for material derivatives, which are not under consideration in the paper, ought to be revisited.

\subsection{Methods of asymptotic analysis}

Nowadays there exist several methods to construct asymptotic expansions of solutions to elliptic boundary value problems in domains with singular perturbations of boundaries. First of all, we mention two methods, namely, the method of mathed asymptotic expansions and the method of compound asymptotic expansions (cf. monographs [16] and 28, respectively), which in general appear to be of the same power. Indeed, based on different asymptotic procedures, the matching procedure and the procedure of of rearrangement of discrepancies, they result in asymptotic expansions which differ at the first sight one from another, but can be readily transformed one into another (we refer to the introductory chapter 2 in 28]). By the way, we silently use this transformation while presenting at the end of Section 3.5 an alternative way of presentation the asymptotic form of the derived solution. The method of compound asymptotics is employed throughout the paper for two reasons. First, the results given in 28. are obtained in relatively general formulation which includes the elliptic systems of partial differential equations not necessarily formally self-adjoint (cf. discussion in the preceding Section 2.1). On the other hand, in the monograph [16] the results are established exclusively for the scalar second-order elliptic equations in the divergence form. Second, the method of compound asymptotic expansions is carefully matched with theory of elliptic problems in domains with conical outlets to infinity, specifically the exterior domains (cf. [[39]; Ch.6], and [35]) while for our purposes this theory is used further to introduce and investigate the polarization matrices in piezoelectricity.

Since the problem under studies is geometrically very specific, i.e., it concerns only one small opening inside of a domain in $\mathbb{R}^{3}$, the other methods of asymptotic analysis can be employed. In Remark 18 (2) we mention the case of piecewise constant coefficients which makes suitable an asymptotic analysis of the equivalent boundary integral equations obtained from fundamental solution (cf. 3] with similar results in elasticity), although no fundamental matrix is known in piezoelectricity. The other possibilities include among others the homogeneization technique relying on the so-called delute limit (see [17, 31, [26] and many others). However, in our opinion, the method of compound asymptotic expansions is still the most appropriate tool in piezoelectricity in order to investigate asymptotic properties of shape functionals.

\subsection{Constitutive relations in piezoelectricity}

Let $\Omega \subset \mathbb{R}^{3}$ be a piezoelectric body with the Lipschitz boundary $\partial \Omega$ and the compact closure $\bar{\Omega}=\Omega \cup \partial \Omega$. Using the matrix/column notation (cf. 24, 33]), we regard the displacement vector $u^{\mathrm{M}}$ as the column $u^{\mathrm{M}}=\left(u_{1}^{\mathrm{M}}, u_{2}^{\mathrm{M}}, u_{3}^{\mathrm{M}}\right)^{\top}$ where $u_{j}^{\mathrm{M}}$ is the projection of $u$ on the $x_{j}$-axis of the fixed Cartesian coordinates system $x=\left(x_{1}, x_{2}, x_{3}\right)^{\top}$ and $\top$ stands for transposition. Together with the electric potential $u^{\mathrm{E}}$, the displacements compose the column $u=\left(u_{1}^{\mathrm{M}}, u_{2}^{\mathrm{M}}, u_{3}^{\mathrm{M}}, u^{\mathrm{E}}\right)^{\top}$ of height 4 . The strain column

$$
\varepsilon^{\mathrm{M}}\left(u^{\mathrm{M}}\right)=\left(\varepsilon_{11}^{\mathrm{M}}, \varepsilon_{22}^{\mathrm{M}}, \varepsilon_{33}^{\mathrm{M}}, \sqrt{2} \varepsilon_{23}^{\mathrm{M}}, \sqrt{2} \varepsilon_{31}^{\mathrm{M}}, \sqrt{2} \varepsilon_{12}^{\mathrm{M}}\right)^{\top}
$$

consists of the Cartesian components $\varepsilon_{j k}^{\mathrm{M}}=\frac{1}{2}\left(\partial_{j} u_{k}^{\mathrm{M}}+\partial_{k} u_{j}^{\mathrm{M}}\right)$ of the strain tensor and takes the form $\varepsilon^{\mathrm{M}}\left(u^{\mathrm{M}}\right)=D^{\mathrm{M}}\left(\nabla_{x}\right) u^{\mathrm{M}}$ where

$$
D^{\mathrm{M}}\left(\nabla_{x}\right)^{\top}=\left(\begin{array}{cccccc}
\partial_{1} & 0 & 0 & 0 & 2^{-1 / 2} \partial_{3} & 2^{-1 / 2} \partial_{2} \\
0 & \partial_{2} & 0 & 2^{-1 / 2} \partial_{3} & 0 & 2^{-1 / 2} \partial_{1} \\
0 & 0 & \partial_{3} & 2^{-1 / 2} \partial_{2} & 2^{-1 / 2} \partial_{1} & 0
\end{array}\right), \nabla_{x}=\left(\begin{array}{c}
\partial_{1} \\
\partial_{2} \\
\partial_{3}
\end{array}\right), \partial_{j}=\frac{\partial}{\partial x_{j}} .
$$


We introduce the column $\varepsilon(u)=\left(\varepsilon^{\mathrm{M}}\left(u^{\mathrm{M}}\right)^{\top}, \varepsilon^{\mathrm{E}}\left(u^{\mathrm{E}}\right)^{\top}\right)^{\top}$ where $\varepsilon^{\mathrm{E}}\left(u^{\mathrm{E}}\right)=\nabla_{x} u^{\mathrm{E}}$ is the electric strain column, taken with the sign minus, and $D\left(\nabla_{x}\right)$ implies a $(9 \times 4)$-matrix of the first-order differential operators,

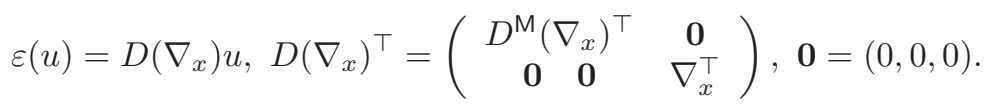

We also assemble the column $\sigma(u)$ of height 9 from the stress column $\sigma^{\mathrm{E}}\left(u^{\mathrm{M}}\right)$ of structure (2.3) and the electric induction column $\sigma^{\mathrm{E}}\left(u^{\mathrm{E}}\right)=\left(\sigma_{1}^{\mathrm{E}}, \sigma_{2}^{\mathrm{E}}, \sigma_{3}^{\mathrm{E}}\right)^{\top}$. In this manner, the constitutive relations of piezoelectricity (see [12, 15, 46])

$$
\sigma^{\mathrm{M}}=A^{\mathrm{MM}} \varepsilon^{\mathrm{M}}-A^{\mathrm{ME}} \varepsilon^{\mathrm{E}}, \sigma^{\mathrm{E}}=A^{\mathrm{EM}} \varepsilon^{\mathrm{M}}+A^{\mathrm{EE}} \varepsilon^{\mathrm{E}}
$$

can be rewritten as follows:

$$
\sigma(u)=A \varepsilon(u)
$$

where the matrix $A$ of size $9 \times 9$,

$$
A=\left(\begin{array}{cc}
A^{\mathrm{MM}} & -A^{\mathrm{ME}} \\
A^{\mathrm{EM}} & A^{\mathrm{EE}}
\end{array}\right)
$$

is formed by the symmetric and positive definite $(6 \times 6)$ - and $(3 \times 3)$-matrices $A^{\mathrm{MM}}$ and $A^{\mathrm{EE}}$, respectively the elastic stiffness matrix and the dielectric permeability matrix, and the blocks $A^{\mathrm{ME}}=\left(A^{\mathrm{EM}}\right)^{\top}$ of piezoelectric moduli. We emphasize that, by its physical nature, the matrix (2.8) is not symmetric provided the $(6 \times 3)$-block $A^{\mathrm{ME}}$ is not null, i.e., the mechanical and electric fields interact.

The state of the piezoelectric body $\Omega$ is described by the mixed boundary value problem

$$
\begin{array}{r}
D\left(-\nabla_{x}\right)^{\top} A(x) D\left(\nabla_{x}\right) u(x)=f(x), x \in \Omega, \\
D(n(x))^{\top} A(x) D\left(\nabla_{x}\right) u(x)=g(x), x \in \Gamma_{\sigma}, \\
u(x)=0, x \in \Gamma_{u}=\partial \Omega \backslash \bar{\Gamma}_{\sigma},
\end{array}
$$

where $n=\left(n_{1}, n_{2}, n_{3}\right)^{\top}$ is the unit vector (column) of the outward normal. On the right hand-side of the equations (2.9) and (2.10), we have the volume forces $f^{\mathrm{M}}=\left(f_{1}^{\mathrm{M}}, f_{2}^{\mathrm{M}}, f_{3}^{\mathrm{M}}\right)^{\top}$ and the surface mechanical loading $g^{\mathrm{M}}=\left(g_{1}^{\mathrm{M}}, g_{2}^{\mathrm{M}}, g_{3}^{\mathrm{M}}\right)^{\top}$ together with the volume $f^{\mathrm{E}}$ and surface $g^{\mathrm{E}}$ electric charges. The Dirichlet conditions (2.11) mean that the body is mechanically clamped over the surface $\Gamma_{u}$ and in contact with an electric conductor. As usually, $f^{\mathrm{E}}=0$ and, if the surface $\Gamma_{\sigma}$ is in contact with a dielectric medium, i.e., vacuum, we are to put $g_{4}^{E}=0$.

\subsection{Solvability of boundary value problem}

Let us assume that $\operatorname{mes}_{2} \Gamma_{u}>0$ and $f \in L^{2}(\Omega)^{4}, g \in L^{2}\left(\Gamma_{\sigma}\right)^{4}$ where $L^{2}(\Xi)$ denote the Lebesque space with the intrinsic inner product $(,) \Xi$ and the superscript 4 indicates the number of components in the vector functions $f$ and $g$. Notice that the subscript is always omitted in our notation for inner products and norms.

The integral identity (cf. 23]) serving for problem (2.9)-(2.11), reads as follows:

$$
Q(u, v ; \Omega):=\left(A D\left(\nabla_{x}\right) u, D\left(\nabla_{x}\right) v\right)_{\Omega}=(f, v)_{\Omega}+(g, v)_{\Gamma_{u}}, v \in \stackrel{\circ}{H}^{1}\left(\Omega ; \Gamma_{u}\right)^{4},
$$

where $\stackrel{\circ}{H}^{1}\left(\Omega ; \Gamma_{u}\right)$ denotes the Sobolev space of functions vanishing at $\Gamma_{u}$. The left-hand side of (2.12) is understood properly provided entries of the matrix $A$ are measurable and uniformly bounded functions in $\Omega$. In addition, for almost all $x \in \Omega$, we assume the symmetry and positivity properties

$$
\begin{aligned}
& A^{\mathrm{MM}}(x)=A^{\mathrm{MM}}(x)^{\top}, A^{\mathrm{ME}}(x)=A^{\mathrm{ME}}(x)^{\top}, A^{\mathrm{EE}}(x)=A^{\mathrm{EE}}(x)^{\top}, \\
& c_{\mathrm{M}}\left|a^{\mathrm{M}}\right|^{2} \leq\left(a^{\mathrm{M}}\right)^{\top} A^{\mathrm{MM}}(x) \leq C_{\mathrm{M}}\left|a^{\mathrm{M}}\right|^{2}, a^{\mathrm{M}} \in \mathbb{R}^{6}, \\
& c_{\mathrm{E}}\left|a^{\mathrm{E}}\right|^{2} \leq\left(a^{\mathrm{E}}\right)^{\top} A^{\mathrm{EE}}(x) \leq C_{\mathrm{E}}\left|a^{\mathrm{E}}\right|^{2}, a^{\mathrm{E}} \in \mathbb{R}^{3},
\end{aligned}
$$


where $c_{\mathrm{M}}, C_{\mathrm{M}}$ and $c_{\mathrm{E}}, C_{\mathrm{E}}$ are positive constants. We emphasize that no positivity restriction is imposed on the piezoelectric moduli in $A^{\mathrm{ME}}$.

Although in the case $A^{\mathrm{ME}} \neq 0$ the sesquilinear form $Q(\cdot, \cdot ; \Omega)$ cannot be an inner product on the Hilbert space $\stackrel{\circ}{H}^{1}\left(\Omega ; \Gamma_{u}\right)^{4}$ due to the wrong sign on $A^{\mathrm{ME}}$ in (2.8), the Lax-Milgram lemma ensures the following assertion because of the formula

$$
Q(u, u ; \Omega)=\left(A^{\mathrm{MM}} D^{\mathrm{M}}\left(\nabla_{x}\right) u^{\mathrm{M}}, D^{\mathrm{M}}\left(\nabla_{x}\right) u^{\mathrm{M}}\right)_{\Omega}+\left(A^{\mathrm{EE}} \nabla_{x} u^{\mathrm{E}}, \nabla_{x} u^{\mathrm{E}}\right)_{\Omega} \geq c\left\|u ; H^{1}(\Omega)\right\|^{2}
$$

caused by the Poincaré inequality for $u^{\mathrm{E}}$ and the Korn inequality for $u^{\mathrm{M}}$ (see [7, 21] and others).

Proposition 1 Under the conditions (2.13), (2.14), the problem (2.12) admits a unique solution $u \in$ $\stackrel{\circ}{H}^{1}\left(\Omega ; \Gamma_{u}\right)^{4}$, and the following estimate is valid:

$$
\left\|u ; H^{1}(\Omega)\right\| \leq c_{\Omega}\left(\left\|f ; L^{2}(\Omega)\right\|+\left\|g ; L^{2}\left(\Gamma_{\sigma}\right)\right\|\right) .
$$

Unfortunately, the problem (2.12) is non variational. Indeed, the energy functional $\mathcal{U}$,

$$
\begin{array}{r}
\mathcal{U}(u ; \Omega)=\frac{1}{2}\left(A D\left(\nabla_{x}\right) u, D\left(\nabla_{x}\right) u\right)_{\Omega}-\mathcal{A}(u ; \Omega), \\
\mathcal{A}(u ; \Omega):=(f, u)_{\Omega}+(g, u)_{\Gamma_{\sigma}},
\end{array}
$$

is but the sum of the mechanical and electric energy functionals

$$
\begin{aligned}
\mathcal{U}^{\mathrm{M}}\left(u^{\mathrm{M}} ; \Omega\right)= & \frac{1}{2}\left(A^{\mathrm{MM}} D^{\mathrm{M}}\left(\nabla_{x}\right) u^{\mathrm{M}}, D^{\mathrm{M}}\left(\nabla_{x}\right) u^{\mathrm{M}}\right)_{\Omega}-\left(f^{\mathrm{M}}, u^{\mathrm{M}}\right)_{\Omega}-\left(g^{\mathrm{M}}, u^{\mathrm{M}}\right)_{\Gamma_{\sigma}}, \\
& \mathcal{U}^{\mathrm{E}}\left(u^{\mathrm{E}} ; \Omega\right)=\frac{1}{2}\left(A^{\mathrm{EE}} \nabla_{x} u^{\mathrm{E}}, \nabla_{x} u^{\mathrm{E}}\right)_{\Omega}-\left(f^{\mathrm{E}}, u^{\mathrm{E}}\right)_{\Omega}-\left(g^{\mathrm{E}}, u^{\mathrm{E}}\right)_{\Gamma_{\sigma}},
\end{aligned}
$$

while a stationary point of (2.16) becomes a solution of the problem (2.12) with the block-diagonal $(9 \times 9)$ matrix $\operatorname{diag}\left\{A^{\mathrm{MM}}, A^{\mathrm{EE}}\right\}$, i.e., the variational problem does not accept an interaction of the mechanical and electric fields (cf. an example in Section 4.4).

It is known (see, e.g., [52]) that the electric enthalpy $\mathcal{E}$,

$$
\begin{array}{r}
\mathcal{E}(u ; \Omega)=\frac{1}{2}\left(A_{(-)} D\left(\nabla_{x}\right) u, D\left(\nabla_{x}\right) u\right)_{\Omega}-\mathcal{R}(u ; \Omega), \\
\mathcal{R}(u ; \Omega)=\left(f^{\mathrm{M}}, u^{\mathrm{M}}\right)_{\Omega}+\left(g^{\mathrm{M}}, u^{\mathrm{M}}\right)_{\Gamma_{\sigma}}-\left(f_{4}^{\mathrm{E}}, u_{4}^{\mathrm{E}}\right)_{\Omega}-\left(g_{4}^{\mathrm{E}}, u_{4}^{\mathrm{E}}\right)_{\Gamma_{\sigma}},
\end{array}
$$

gives rise to the variational formulation of the piezoelectricity problem

$$
Q_{(-)}(u, v ; \Omega):=\left(A_{(-)} D\left(\nabla_{x}\right) u, D\left(\nabla_{x}\right) v\right)_{\Omega}=\mathcal{R}(v ; \Omega), v \in \stackrel{\circ}{H}^{1}\left(\Omega ; \Gamma_{\sigma}\right)^{4},
$$

where the matrix $A_{(-)}$is composed from blocks in (2.8) as follows

$$
A_{(-)}=\left(\begin{array}{cc}
A^{\mathrm{MM}} & A^{\mathrm{ME}} \\
A^{\mathrm{EM}} & -A^{\mathrm{EE}}
\end{array}\right)
$$

The matrix (2.23), in contrast to the matrix $A$, is symmetric, however, neither matrix (2.23), nor the quadratic form on the left-hand side of (2.22) is positive definite. Thus, a solution $u \in \stackrel{H^{1}}{ }\left(\Omega ; \Gamma_{\sigma}\right)^{4}$ is a stationary point of the functional (2.20) but $u$ cannot be any minimizer of the electric enthalpy $\mathcal{E}(u ; \Omega)$.

The integral identity (2.22) with the test function $v_{(-)}=\left(v_{1}^{\mathrm{M}}, v_{2}^{\mathrm{M}}, v_{3}^{\mathrm{M}},-v^{\mathrm{E}}\right)$ transforms into the problem (2.12). The inverse transformation is also available. These facts prove that the problem (2.22) inherits the unique solvability from (2.12) in Proposition 1 .

Remark 2 The integral identity is formally obtained by the multiplying system (2.9) with $v$ scalarly and integrating by parts. Using $v_{(-)}$as the multiplier, one arrives at (2.22). This explains the equivalency of the problems. 
Remark 3 In Section 3.3 we shall outline a different way to modify the piezoelectricity problem in order to study properties of the mechanical and electric fields on the base of known results. This approach is related to studies [31], [4], and others, on variational formulations of elliptic problems describing processes in media with complex material coefficients (phase changes due to material properties). In fact we need this technique only for one reason, to maintain the so-called polynomial property [34, 36], thus the results [31], [4] are not applied in the paper. We recall that the polynomial property allows to describe all required attributes of the exterior boundary value problem of piezoelectricity by simple algebraic calculations (cf. review [35]).

The electric enthalpy is but the difference of elastic energy (2.18) and electric energy (2.19). Expression (2.17) implies the external work. Being the difference of the mechanical and electric external works, the component $\mathcal{R}(u ; \Omega)$ of the electric enthalpy has no physical meaning as a whole. Nevertheless, in Section 4.2 we shall observe that asymptotic formulae for $\mathcal{E}(u ; \Omega)$ become meaningful while the analogous formulae for $\mathcal{U}(u ; \Omega)$ look rather queer.

\subsection{Structure of the paper}

In Section 3 the asymptotic analysis of the piezoelectricity problem for the body $\Omega(h)$ with a small void $\overline{\omega_{h}}$ is performed (see (3.1)). The applied here asymptotic procedure [28, Ch.4] requires for introduction of an intrinsic integral characteristics of the void $\overline{\omega_{1}}$ in the homogeneous piezoelectric space $\mathbb{R}^{3}$, the polarization matrix $M\left(A^{0}, \omega\right)$ of size $9 \times 9$ (see formulae (3.38)-(3.40) $)$. Theorem 12 establishes general properties of the polarization matrix, see also (4.69) for the case of weak interaction between mechanical and electric fields. The polarization matrix appears in the asymptotic expansion of the boundary layer term at infinity that also permits in Section 3.5 to complete the asymptotic ansatz of the solution to the piezoelectricity problem in $\Omega(h)$. The asymptotics constructed in Section 3 is justified in Section 4.1 In Section 4.2 the asymptotics of the energy and electric enthalpy functionals are analysed, while in Section 4.3 rather arbitrary shape functional is considered and the corresponding adjoint state is detected. The paper is completed by inquiring into a piezoelectric body with a weak interaction of the mechanical and electric fields. All asymptotic formulae derived in the paper are made more explicit in such a case due to the fact that for pure electricity and pure elasticity the polarization matrices are known explicitly for many canonical shapes (see, respectively, [48, [53, 25, 3] and others).

\section{Asymptotic analysis}

\subsection{The problem with an interior singular perturbation in the domain}

Let $\omega$ be an open set in $\mathbb{R}^{3}$ with a Lipschitz boundary and a compact closure. We assume that both $\Omega$ and $\omega$ contain the coordinate origin $\mathcal{O}$. Given a small dimensionless parameter $h \in\left(0, h_{0}\right]$, we introduce the sets

$$
\omega_{h}=\left\{x: \xi:=h^{-1} x \in \omega\right\}, \Omega(h)=\Omega \backslash \bar{\omega}_{h} .
$$

The bound $h_{0}>0$ is chosen such that $\bar{\omega}_{h} \subset \Omega$ for $h \in\left(0, h_{0}\right]$. By rescaling, we reduce a characteristic size of $\Omega$ and $\omega$ to the unit and make the coordinates $x$ and $\xi$ dimensionless.

Supposing $\Omega(h)$ to be a connected set, we consider the piezoelectricity problem in the domain $\Omega(h)$, namely,

$$
\begin{array}{r}
D\left(-\nabla_{x}\right)^{\top} A(x) D\left(\nabla_{x}\right) u^{h}(x)=f(x), x \in \Omega(h), \\
D(n(x))^{\top} A(x) D\left(\nabla_{x}\right) u^{h}(x)=g(x), x \in \Gamma_{\sigma}, \\
D\left(n^{h}(x)\right)^{\top} A(x) D\left(\nabla_{x}\right) u^{h}(x)=0, x \in \partial \omega_{h}, \\
u^{h}(x)=0, x \in \Gamma_{u} .
\end{array}
$$


In (3.4), $n^{h}$ stands for the outward normal on $\partial \omega_{h}$. Since the Neumann conditions are imposed on the boundary of $\omega_{h}$, there is no traction on $\partial \omega_{h}$ and the opening $\overline{\omega_{h}}$ is filled with a dielectric medium. This problem, of course, ought to be reformulated as either integral identity (2.12), or (2.22) in the function space $\stackrel{\circ}{H}^{1}\left(\Omega(h) ; \Gamma_{u}\right)^{4}$, hence

$$
Q\left(u^{h}, v^{h} ; \Omega(h)\right)=\left(f, v^{h}\right)_{\Omega(h)}+\left(g, v^{h}\right)_{\Gamma_{\sigma}}, v^{h} \in \stackrel{\circ}{H}^{1}\left(\Omega(h) ; \Gamma_{u}\right)^{4} .
$$

Proposition 1 remains valid for the problem (3.6) in the domain $\Omega(h)$.

For $h=0$, the opening $\omega_{h}$ disappears and the singularly perturbed problem (3.2)-(3.5) becomes the original problem (2.9)-(2.11). In order to describe the behavior of the solution $u^{h} \in \stackrel{\circ}{H}^{1}\left(\Omega(h) ; \Gamma_{u}\right)^{4}$ as $h \rightarrow+0$, we have to assume an additional smoothness of the matrix $A$, for example, in the ball $\mathbb{B}_{R}=\{x:|x|<R\}$ the inclusion

$$
A \in C^{2, \alpha}\left(\overline{\mathbb{B}}_{R}\right)^{9 \times 9}
$$

is valid, where $C^{k, \alpha}(\Xi)$ is the Hölder space with the standard norm

$$
\left\|v ; C^{k, \alpha}(\Xi)\right\|=\sum_{j=1}^{k} \sup _{x \in \Xi}\left|\nabla_{x}^{j} v(x)\right|+\sup _{x, y \in \Xi}|x-y|^{-\alpha}\left|\nabla_{x}^{k} v(x)-\nabla_{y}^{k} v(y)\right|
$$

and $\nabla_{x}^{k} v$ denotes the family of all order $k$ derivatives of $v$. Since the matrix differential operator

$$
L\left(x, \nabla_{x}\right)=D\left(-\nabla_{x}\right)^{\top} A(x) D\left(\nabla_{x}\right)
$$

is elliptic (see Section 3.3 below), a solution $u \in H^{1}\left(\mathbb{B}_{R}\right)^{4}$ of system (2.9) in $\mathbb{B}_{R}$ with the right-hand side

$$
f \in C^{0, \alpha}\left(\mathbb{B}_{R}\right)^{4}, \alpha \in(1 / 2,1),
$$

falls into the space $C^{2, \alpha}\left(\mathbb{B}_{R^{\prime}}\right)^{4}$ for any $R^{\prime} \in(0, R)$. This fact is due to local estimates of solutions to elliptic systems [1]. Note that (3.9) provides the estimate

$$
|f(x)-f(0)| \leq c|x|^{\alpha}, x \in \mathbb{B}_{R} .
$$

We also need the Taylor formula

$$
\left|u(x)-d(x) a-D(x)^{\top} \varepsilon^{0}-U(x)\right| \leq c|x|^{2+\alpha}, x \in \mathbb{B}_{R^{\prime}},
$$

where $D(x)^{\top}$ is the matrix in (2.5) under the substitution $\nabla_{x} \mapsto x$,

$$
\varepsilon^{0}=D\left(\nabla_{x}\right) u(0) \in \mathbb{R}^{9},
$$

$d(x) a$ with $a \in \mathbb{R}^{7}$ implies a rigid motion in the mechanical component and a constant potential in the electric one,

$$
d(x)=\left(\begin{array}{ccc}
d^{\mathrm{M}}(x) & 0 \\
\mathbf{0} & \mathbf{0} & 1
\end{array}\right), d^{\mathrm{M}}(x)=\left(\begin{array}{cccccc}
1 & 0 & 0 & 0 & -2^{-1 / 2} x_{3} & 2^{-1 / 2} x_{2} \\
0 & 1 & 0 & 2^{-1 / 2} x_{3} & 0 & -2^{-1 / 2} x_{1} \\
0 & 0 & 1 & -2^{-1 / 2} x_{2} & 2^{-1 / 2} x_{1} & 0
\end{array}\right)
$$

We emphasize a similarity of the matrices $D^{\mathrm{M}}(x)^{\top}$ and $d^{\mathrm{M}}(x)$. Finally, $U$ in (3.11) is a quadratic term, i.e.,

$$
U(t x)=t^{2} U(x), t>0, x \in \mathbb{R}^{3} .
$$


Remark 4 The factor $\sqrt{2}$ is present in the strain column (2.3) in order to equalize the natural norms for tensors of rank 2 with the norms of corresponding columns of height 6 . As a result, an orthogonal transformation of the Cartesian coordinate system $x$ implies the orthogonal transformations for all columns introduced to replace tensors (see, e.g., [33, Ch.2]). By the factor $2^{-1 / 2}$ in (3.13), we also achieve the relations

$$
\begin{aligned}
& D\left(\nabla_{x}\right) D(x)^{\top}=\mathbb{I}_{9 \times 9}, \quad D\left(\nabla_{x}\right) d(x)=\mathbb{O}_{9 \times 7}, \\
& \left.d\left(\nabla_{x}\right)^{\top} d(x)\right|_{x=0}=\mathbb{I}_{7 \times 7},\left.\quad d\left(\nabla_{x}\right)^{\top} D(x)^{\top}\right|_{d=0}=\mathbb{O}_{7 \times 9},
\end{aligned}
$$

where $\mathbb{I}_{n \times n}$ and $\mathbb{O}_{m \times n}$ stand for the unit and null matrices of size $n \times n$ and $m \times n$, respectively. Notice that (3.12) follows from the first couple of the relations (3.15) and our way to write the Taylor formula.

By (3.7), we particularly obtain

$$
A(x)=A^{0}+\sum_{j=1}^{3} x_{j} A^{j}+\widetilde{A}(x),\left|\widetilde{A}_{p q}(x)\right| \leq c|x|^{2}, x \in \mathbb{B}_{R},
$$

with the constant $(9 \times 9)$-matrices $A^{j}$ so that matrix (3.8) of differential operator gets the decomposition

$$
L\left(x, \nabla_{x}\right)=L^{0}\left(\nabla_{x}\right)+L^{\prime}\left(x, \nabla_{x}\right)+\widetilde{L}\left(x, \nabla_{x}\right) .
$$

Inserting the Taylor formula for $u$ into the equation (2.9) and using (3.16) yield

$$
L^{0}\left(\nabla_{x}\right) U(x)-\sum_{j=1}^{3} D\left(e_{j}\right)^{\top} A^{j} \varepsilon^{0}=f(0) .
$$

Here $e_{j}=\left(\delta_{j, 1}, \delta_{j, 2}, \delta_{j, 3}\right)^{\top}$. Since $U$ is quadratic in $x$ (see (3.14) $)$, the first term on the left hand-side is independent of $x$.

Remark 5 To guarantee formulae (3.10) and (3.11) with $\alpha \in(0,1 / 2)$, we could assume $f \in H^{2}\left(\mathbb{B}_{R}\right)^{4}$ while deriving $u \in H^{4}\left(\mathbb{B}_{R^{\prime}}\right)^{4}$ from local estimates for solutions of elliptic systems (see [1]). This is due to the Sobolev embedding theorem $H^{l+2} \subset C^{l, \alpha}$ in $\mathbb{R}^{3}$ for any $\alpha \in(0,1 / 2)$. However, in Theorem 19 and Remark 20 we shall see that we really need $\alpha>1 / 2$. The latter requires, for example, $f \in H^{3}\left(\mathbb{B}_{R}\right)^{4}$, and, therefore, we prefer here to use the Hölder scale.

\subsection{The asymptotic ansatz}

Based on general results in [28] on the asymptotic structure of solutions to elliptic boundary value problems in a domain with singular perturbations of the boundary, we accept the following asymptotic ansatz for the solution $u^{h}$ of problem (3.2)-3.5):

$$
u^{h}(x)=u(x)+\chi(x)\left(h w^{1}(\xi)+h^{2} w^{2}(\xi)\right)+h^{3} \mathbf{u}(x)+\ldots
$$

Here $u$ is a solution of the limit problem (2.9)-(2.11), $w^{1}$ and $w^{2}$ are terms of the boundary layer type, and $\mathbf{u}$ is the main regular corrector. The boundary layer terms are treated in Sections 3.3 and 3.4 . respectively, and the regular corrector in Section 3.5 below. The cut-off function $\chi \in C_{c}^{\infty}(\Omega)$ is equal to one in the ball $\mathbb{B}_{R / 3}$ and null outside $\mathbb{B}_{2 R / 3}$ so that, now, we fix $h_{0}>0$ such that $\omega_{h} \subset \mathbb{B}_{R / 3}$ for $h \in\left(0, h_{0}\right]$.

Remark 6 The boundary layer solutions $w^{1}$ and $w^{2}$ are constructed in Sections 3.3 and 3.4 respectively, along with their decompositions at infinity. In Section 3.5 the main terms of the decompositions, compose the right-hand side of a problem of type (2.9)-(2.11) for the regular solution $\mathbf{u}$. 
In view of (3.1), the coordinate dilation $x \mapsto \xi=h^{-1} x$ removes the boundary $\partial \Omega$ close to infinity and the formal limit passage $h \rightarrow+0$ makes the exterior domain $\Xi=\mathbb{R}^{3} \backslash \bar{\omega}$ from the nucleated domain $\Omega(h)$. Moreover, the decomposition (3.17) yields

$$
L\left(x, \nabla_{x}\right)=L\left(h \xi, h^{-1} \nabla_{\xi}\right)=h^{-2} L^{0}\left(\nabla_{\xi}\right)+h^{-1} L^{\prime}\left(\xi, \nabla_{\xi}\right)+\ldots
$$

Similarly, for the Neumann boundary operator $N^{h}\left(x, \nabla_{x}\right)$ on the left hand-side of (3.4), we have

$$
N^{h}\left(x, \nabla_{x}\right)=h^{-1} N^{0}\left(\xi, \nabla_{\xi}\right)+h^{0} N^{\prime}\left(\xi, \nabla_{\xi}\right)+\ldots
$$

where

$$
N^{0}\left(\xi, \nabla_{\xi}\right)=D\left(n^{\omega}(\xi)\right)^{\top} A^{0} D\left(\nabla_{x}\right), N^{\prime}\left(\xi, \nabla_{\xi}\right)=D\left(n^{\omega}(\xi)\right)^{\top} \sum_{j=1}^{3} \xi_{j} A^{j} D\left(\nabla_{\xi}\right),
$$

and $n^{\omega}$ is the unit vector of the outward normal on $\partial \omega$.

Let us derive the exterior boundary value problems for $w^{1}$ and $w^{2}$. First, we insert the ansatz (3.19) into (3.2), make use of the expansion (3.21), and collect coefficients written in the fast variables $\xi$ for similar powers of the small parameter $h$. As a result, we obtain systems of differential equations in $\Xi$ for $w^{1}$ and $w^{2}$ (see (3.23) and (3.24) below). Second, we calculate the discrepancy left by the leading asymptotic term $u(x)$ in the boundary conditions (3.4). Namely, by means of (3.11), (3.21), we derive that

$$
N^{h}\left(x, \nabla_{x}\right) u(x)=D\left(n^{\omega}(\xi)\right)^{\top}\left(A^{0}+h \sum_{j=1}^{3} \xi_{j} A^{j}\right) \varepsilon^{0}+h N^{0}\left(\xi, \nabla_{\xi}\right) U(\xi)+\ldots
$$

Finally, we write the problems

$$
\begin{aligned}
& L^{0}\left(\nabla_{\xi}\right) w^{1}(\xi)=0, \xi \in \Xi, \\
& N^{0}\left(\xi, \nabla_{\xi}\right) w^{1}(\xi)=-D\left(n^{\omega}(\xi)\right)^{\top} A^{0} \varepsilon^{0}, \xi \in \partial \omega,
\end{aligned}
$$

and

$$
\begin{aligned}
& L^{0}\left(\nabla_{\xi}\right) w^{2}(\xi)=-L^{\prime}\left(\xi, \nabla_{\xi}\right) w^{1}(\xi), \xi \in \Xi \\
& N^{0}\left(\xi, \nabla_{\xi}\right) w^{2}(\xi)=-N^{\prime}\left(\xi, \nabla_{\xi}\right) w^{1}(\xi)-N^{\prime}\left(\xi, \nabla_{\xi}\right) D(\xi)^{\top} \varepsilon_{0}-N^{0}\left(\xi, \nabla_{\xi}\right) U(\xi), \quad \xi \in \partial \omega .
\end{aligned}
$$

\subsection{The exterior problem in piezoelectricity}

The polynomial property [34, 35] of a formally self-adjoint system of differential equations delivers plenty of results for the exterior boundary value problem in $\Xi$ such as the ellipticity, the solvability, asymptotic expansions of solutions, and intrinsic integral characteristics, i.e. the polarization matrices (see [39, Ch.6], 35, 37] and [40 in shape optimization). As it has been mentioned, the piezoelectricity system (2.9) is not formally self-adjoint, however, introducing the imaginary potential $i u_{4}^{\mathrm{E}}$ (see 35, Example 1.13]) and the column $u_{(i)}=\left(u_{1}^{\mathrm{M}}, u_{2}^{\mathrm{M}}, u_{3}^{\mathrm{M}}, i u_{4}^{\mathrm{E}}\right)^{\top}$ brings the sesquilinear form

$$
q_{(i)}\left(u_{(i)}, v_{(i)} ; \Xi\right)=\left(A_{(i)}^{0} D\left(\nabla_{\xi}\right) u_{(i)}, D\left(\nabla_{\xi}\right) v_{(i)}\right) \Xi
$$

where $i$ is the imaginary unit and $A_{(i)}^{0}$ stands for modified matrix (2.23),

$$
A_{(i)}^{0}=\left(\begin{array}{cc}
A^{0 \mathrm{MM}}, & i A^{0 \mathrm{ME}} \\
i A^{0 \mathrm{EM}}, & A^{0 \mathrm{EE}}
\end{array}\right)=A_{(R e)}^{0}+i A_{(I m)}^{0},
$$

while both $A_{(R e)}^{0}$ and $A_{(I m)}^{0}$ are real symmetric and $A_{(R e)}^{0}$ is positive definite. The sesquilinear form (3.25) is not Hermitian in the case $A^{0 \mathrm{ME}} \neq \mathbb{O}_{6 \times 3}$, but it enjoys the polynomial property [34, 36, 35]:

$$
q_{(i)}\left(u_{(i)}, u_{(i)} ; \Upsilon\right)=\left.0 \Longleftrightarrow u_{(i)} \in \mathcal{P}\right|_{\Upsilon}
$$


where $\Upsilon$ is any domain in $\mathbb{R}^{3}$ and $\mathcal{P}=\left\{p: p(x)=d(x) a, a \in \mathbb{C}^{7}\right\}$ is a polynomial subspace of dimension 7 generated by the matrix in (3.13).

The above observations made in [36, 35] and the investigation scheme [39, Ch.6] provide all results we formulate below with exception for the polarization matrix and here the most attention is paid to this integral characteristics of the opening $\bar{\omega}$ in the homogeneous piezoelectric space.

Let $V_{0}^{1}(\Xi)$ be the Kondratiev space $\left[20\right.$ obtained by the completion of the linear space $C_{c}^{\infty}(\bar{\Xi})$ (infinitely differentiable functions with compact supports) with respect to the Dirichlet integral norm $\left\|\nabla_{\xi} w ; L^{2}(\Xi)\right\|$. Applying the one-dimensional Hardy inequality in the radial variable $\rho=|\xi|$, we use the equivalent norm

$$
\left\|w ; V_{0}^{1}(\Xi)\right\|=\left(\left\|\nabla_{\xi} w ; L^{2}(\Xi)\right\|^{2}+\left\|\rho^{-1} w ; L^{2}(\Xi)\right\|^{2}\right)^{1 / 2} .
$$

The problem (3.23) with the right-hand side $g \in L^{2}(\partial \omega)^{4}$ in the Neumann boundary conditions can be reformulated as the integral identity, similarly to (2.12)

$$
\left(A^{0} D\left(\nabla_{\xi}\right) w, D\left(\nabla_{\xi}\right) v\right)_{\Xi}=(g, v)_{\partial \omega}, v \in V_{0}^{1}(\Xi)^{4} .
$$

Proposition 7 For any $g \in L^{2}(\partial \omega)^{4}$, the problem (3.29) has a unique solution $w \in V_{0}^{1}(\Xi)^{4}$ and the estimate $\left\|w ; V_{0}^{1}(\Xi)\right\| \leq c\left\|g ; L^{2}(\partial \omega)\right\|$ is valid.

Although $\partial \omega$ and $g$ are not smooth, the solution $w$ in Proposition 7 is infinitely differentiable outside of any neighborhood $\mathcal{V}$ of the set $\bar{\omega}$ (recall the local estimates in 1 mentioned above). To describe the behavior of $w(\xi)$ as $\rho \rightarrow \infty$, we introduce the fundamental matrix $\Phi(x)$ of size $4 \times 4$ for the operator $L^{0}\left(\nabla_{\xi}\right)$ in $\mathbb{R}^{3}$ (see [11, 13]). This matrix is positive homogeneous of degree -1 , namely,

$$
\Phi(t \xi)=t^{-1} \Phi(\xi), t>0, \xi \in \mathbb{R}^{3} \backslash\{0\} .
$$

The next assertion is due to [20, 30] (see also [4] and, e.g., [39, Ch.6]).

Proposition 8 The solution $w \in V_{0}^{1}(\Xi)^{4}$ of the problem (3.29) admits the asymptotic form

$$
\begin{gathered}
w(\xi)=\left(d\left(-\nabla_{\xi}\right)^{\top} \Phi(\xi)^{\top}\right)^{\top} a+\left(D\left(-\nabla_{\xi}\right) \Phi(\xi)^{\top}\right)^{\top} b+\widetilde{w}(\xi), \\
\left|\nabla_{\xi}^{k} \widetilde{w}(\xi)\right| \leq c_{k} \rho^{-3-k}, k \in \mathbb{N}_{0}=\{0,1,2, \ldots\}, \quad \xi \in \mathbb{R}^{3} \backslash \mathcal{V},
\end{gathered}
$$

where $a \in \mathbb{R}^{7}$ and $b \in \mathbb{R}^{9}$ while $|a|+|b| \leq c\left\|g ; L^{2}(\partial \omega)\right\|$.

Remark 9 Formulae (3.31)-(3.32) can be derived from the integral representation of the solution $w$ through the fundamental matrix (3.30). In this way decomposition (3.32) is obtained from the Taylor formula in inverted variables $\xi|\xi|^{-2}$. Observe that the columns of matrices $D(x)^{\top}$ and $d(x)$ in (2.5) and (3.13) form a basis in the linear subspace of dimension 16 of columns linearly dependent on variables $x=$ $\left(x_{1}, x_{2}, x_{3}\right)$. We emphasize that the matrix notation of elasticity relations combined with the polynomial property allow us to write the complete decomposition of $w(\xi)$ for $|\xi| \rightarrow \infty$ in a condensed and convenient form for further applications.

Remark 10 Formula (3.31) contains the matrices $d$ and $D$ in (3.13) and (2.5). Let $d^{1}(\xi), \ldots, d^{7}(\xi)$ be columns of $d(\xi)$ and let $D_{1}(\xi), \ldots, D_{9}(\xi)$ be strings of $D(\xi)$. Then we rewrite (3.31) in the form of strings

$$
w(\xi)^{\top}=\sum_{j=1}^{7} a_{j} d^{j}\left(-\nabla_{\xi}\right)^{\top} \Phi(\xi)^{\top}+\sum_{k=1}^{9} b_{k} D_{k}\left(-\nabla_{\xi}\right) \Phi(\xi)^{\top}+\widetilde{w}(\xi)^{\top} .
$$

Therefore, the asymptotic terms detached in (3.31) are but a linear combination of columns of the fundamental matrix $\Phi(\xi)$ (with the coefficients $a_{1}, a_{2}, a_{3}$ and $a_{7} ; c f .(\sqrt{3.13})$ ) and of the first-order derivatives of the columns (with the coefficients $a_{4}, a_{5}, a_{6}$ and $b_{1}, \ldots, b_{9}$ ). 
The columns $d^{1}, \ldots, d^{7}$ satisfy the homogeneous problem (3.23). However, the columns are not in the weighted space $V_{0}^{1}(\Xi)^{4}$ by the lack of their decay rate and, hence, $d^{j}(\xi)$ are not solutions of the homogeneous $(g=0)$ problem (3.29) in Proposition 8. According to the general method [29] such solutions are used to compute the coefficients in the asymptotic expansion (3.31). We are going to use this method twice. First, we observe that the right-hand side $g$ in (3.23) verifies the orthogonality conditions

$$
\int_{\partial \omega} d(\xi)^{\top} g(\xi) d s_{\xi}=0 \in \mathbb{R}^{7}
$$

Indeed, by (3.15), we get

$$
\left(\int_{\partial \omega} d(\xi)^{\top} g(\xi) d s_{\xi}\right)^{\top}=-\left(A^{0} \varepsilon^{0}\right)^{\top} \int_{\partial \omega} D\left(n^{\omega}(\xi)\right) d(\xi) d s_{\xi}=-\left(A^{0} \varepsilon^{0}\right)^{\top} \int_{\omega} D\left(\nabla_{\xi}\right) d(\xi) d \xi=0 .
$$

Proposition 11 Under orthogonality condition (3.33), the column $a \in \mathbb{R}^{7}$ in (3.31) vanishes.

The proof is commented in Remark 16.

Let $W^{j} \in V_{0}^{1}(\xi)^{4}$ be a solution to the problem (3.29) with the specific right-hand side

$$
g^{j}(\xi)=-D\left(n^{\omega}(\xi)\right)^{\top} A^{0} \mathbf{e}_{j} ;
$$

here $j=1, \ldots, 9, \mathbf{e}_{j}=\left(\delta_{j, 1}, \ldots, \delta_{j, 9}\right)^{\top}$ is the unit column in $\mathbb{R}^{9}$, and $\delta_{j, k}$ stands for the Kronecker symbol. Recalling the problem (3.23) for the boundary layer term $w^{1}$, we see that

$$
w^{1}(\xi)=W(\xi) \varepsilon^{0}
$$

with the $(4 \times 9)$-matrix function $W$ composed from the columns $W^{1}, \ldots, W^{9}$ of height 4 ,

$$
W=\left(W^{1}, \ldots, W^{9}\right) .
$$

By Proposition 11 and the relation (3.34), we conclude the expansions

$$
W^{j}(\xi)^{\top}=\sum_{p=1}^{9} M_{j p} D_{p}\left(\nabla_{\xi}\right) \Phi(\xi)^{\top}+\widetilde{W}^{j}(\xi)^{\top}
$$

where the remainders $\widetilde{W}^{j}(\xi)$ obey the estimates (3.32). The coefficients $M_{j p}$ in (3.38) form the matrix of size $9 \times 9$

$$
M=M\left(A^{0}, \omega\right)
$$

which, in the analogy with [53, 38, 44] and others, is called the polarization matrix of the opening $\omega$ in the homogeneous piezoelectric space.

As in Section 2.4 our study of general properties of (3.39) relies on both formulations (2.12) and (2.22) of the piezoelectricity problem. Hence, we have to perform the same sign changes as in (2.23),

$$
M=\left(\begin{array}{cc}
M^{\mathrm{MM}} & M^{\mathrm{ME}} \\
M^{\mathrm{EM}} & M^{\mathrm{EE}}
\end{array}\right) \mapsto M_{(=)}=\left(\begin{array}{cc}
M^{\mathrm{MM}} & -M^{\mathrm{ME}} \\
M^{\mathrm{EM}} & -M^{\mathrm{EE}}
\end{array}\right) .
$$

Theorem 12 Entries of the modified polarization matrix $M_{(=)}$satisfy the relation

$$
\left(M_{(=)}\right)_{j p}=-Q_{(-)}^{0}\left(W^{j}, W^{p} ; \Xi\right)-\left(A_{(-)}^{0}\right)_{j p} m e s_{3} \omega, j, p=1, \ldots, 9,
$$

where $Q_{(-)}^{0}$ is the quadratic form in (2.22) with the matrix $A_{(-)}^{0}=A_{(-)}(0)$ (see (2.23) and (3.16)). 
Proof. By (3.35) and (3.15), the sum $\mathcal{W}^{j}(\xi)=D_{j}(\xi)^{\top}+W^{j}(\xi)$ verifies the homogeneous problem (3.23). In the method [29] these solutions play the same role as it was registered for the columns $d^{1}, \ldots, d^{7}$ above Proposition 11] We underline that the vector function

$$
\mathcal{W}_{(-)}^{j}=\left(\mathcal{W}_{1}^{j \mathrm{M}}, \mathcal{W}_{2}^{j \mathrm{M}}, \mathcal{W}_{3}^{j \mathrm{M}},-\mathcal{W}^{j \mathrm{E}}\right)^{\top}
$$

verifies a homogeneous boundary value problem which is formally adjoint for (3.23) and involves the differential operators $L_{(\mathrm{T})}^{0}$ and $N_{(\mathrm{T})}^{0}$ constructed from $L^{0}$ and $N^{0}$ in (3.17) and (3.22), respectively, by replacing $A^{0}$ with the transposed matrix $\left(A^{0}\right)^{\top}$. Clearly, $L_{(\top)}^{0}\left(\nabla_{\xi}\right)=L^{0}\left(\nabla_{\xi}\right)^{*}$ is the formally adjoint for the differential operator $L^{0}\left(\nabla_{\xi}\right)$.

We insert $W^{j}$ and $\mathcal{W}_{(-)}^{p}$ into the Green formula written for the truncated domain $\Xi_{R}=\Xi \cap \mathbb{B}_{R}$ and choose the radius of the ball $\mathbb{B}_{R}=\{\xi:|\xi|<R\}$ such that the sphere $\mathbb{S}_{R}=\partial \mathbb{B}_{R}$ envelopes the set $\bar{\omega}$. We have

$$
\left(L^{0} W^{j}, \mathcal{W}_{(-)}^{p}\right) \Xi_{R}+\left(N^{0} W^{j}, \mathcal{W}_{(-)}^{p}\right) \partial \omega \cup \mathbb{S}_{R}=\left(W^{j}, L_{(\top)}^{0} \mathcal{W}_{(-)}^{p}\right)_{\Xi_{R}}+\left(W^{j}, N_{(\top)}^{0} \mathcal{W}_{(-)}^{p}\right)_{\partial \omega \cup \mathbb{S}_{R}} .
$$

Since $L^{0} \mathcal{W}^{j}=0$ provides $L_{(\top)}^{0} \mathcal{W}_{(-)}^{j}=0$, the integrals over $\Xi_{R}$ in (3.43) vanish. Furthermore, $N_{(\top)}^{0}\left(\xi, \nabla_{\xi}\right) \mathcal{W}_{(-)}^{p}(\xi)=$ $0, \xi \in \partial \omega$. Thus, (3.43) converts into

$$
\left(N^{0} W^{j}, \mathcal{W}_{(-)}^{p}\right)_{\partial \omega}=\left(W^{j}, N_{(\top)}^{0} \mathcal{W}_{(-)}^{p}\right)_{\mathbb{S}_{R}}-\left(N^{0} W^{j}, \mathcal{W}_{(-)}^{p}\right)_{\mathbb{S}_{R}}
$$

where $N^{0}\left(\xi, \nabla_{\xi}\right)=D\left(|\xi|^{-1} \xi\right)^{\top} A^{0} D(\xi)$ on the sphere $\mathbb{S}_{R}$.

Taking into account the estimates (3.32) for $\widetilde{W}^{j}$ and the concomitant estimates $\left|\nabla_{\xi}^{k} W^{p}(\xi)\right| \leq$ $c_{p} \rho^{-1-k}$, we obtain that the right-hand side $I_{\text {right }}^{j p}$ of (3.43) satisfies

$$
I_{\text {right }}^{j p}=\left(\Sigma^{j}, N_{(\top)}^{0} D_{p(-)}^{\top}\right)_{\mathbb{S}_{R}}+O\left(R^{-1}\right)
$$

where $\Sigma^{j}$ means the asymptotic term detached in (3.38) and $D_{p(-)}(\xi)^{\top}$ is a column of the matrix $D(\xi)^{\top}$ transformed according to (3.35). Understanding integrals over the ball $\mathbb{B}_{R}$ in the framework of the theory of distributions and using the Green formula, we obtain

$$
\begin{aligned}
I_{\text {right }}^{j p} & =\left(L^{0} \Sigma^{j}, D_{p(-)}^{\top}\right)_{\mathbb{B}_{R}}-\left(\Sigma^{j}, L_{(\top)}^{0} D_{p(-)}^{\top}\right)_{\mathbb{B}_{R}}+O\left(R^{-1}\right) \\
& =\sum_{q=1}^{9} M_{j q} \int_{\mathbb{B}_{R}} D_{p(-)}(\xi) D_{q}\left(\nabla_{\xi}\right)^{\top} \delta(\xi) d \xi+O\left(R^{-1}\right) \\
& =\left.\sum_{q=1}^{9} M_{j q} D_{q}\left(-\nabla_{\xi}\right) D_{p(-)}(\xi)^{\top}\right|_{\xi=0}+O\left(R^{-1}\right) \\
& =\left\{\begin{array}{lll}
-M_{j p} & \text { for } & p=1, \ldots, 6, \\
M_{j p} & \text { for } & p=7,8,9
\end{array}\right\}+O\left(R^{-1}\right)=-\left(M_{(=)}\right)_{j p}+O\left(R^{-1}\right) .
\end{aligned}
$$

Here we have used that, first, $D_{p(-)}(\xi)$ is linear in $\xi$ and, therefore, $L_{(\top)}^{0} D_{p(-)}^{\top}=0$ and, second,

$$
L^{0}\left(\nabla_{\xi}\right) \Sigma^{j}(\xi):=\sum_{q=1}^{9} M_{j q} L^{0}\left(\nabla_{\xi}\right)\left(D_{q}\left(-\nabla_{\xi}\right) \Phi(\xi)^{\top}\right)^{\top}=\sum_{q=1}^{9} M_{j q} D_{q}\left(-\nabla_{\xi}\right)^{\top} \delta(\xi)
$$

caused by the formula $L^{0}\left(\nabla_{\xi}\right) \Phi(\xi)=\delta(\xi) \mathbb{I}_{4 \times 4}$, i.e., by the definition of the fundamental matrix $\Phi$.

Let us process the left-hand side $I_{\text {left }}^{j p}$ of (3.43). Again integrating by parts, this time in the domains $\Xi$ and $\omega$, it follows that

$$
\begin{aligned}
I_{\text {left }}^{j p} & =\left(N^{0} W^{j}, W_{(-)}^{p}\right)_{\partial \Xi}-\left(N^{0} D_{j}^{\top}, D_{p(-)}^{\top}\right)_{\partial \omega} \\
& =Q^{0}\left(W^{j}, W_{(-)}^{p} ; \Xi\right)+Q^{0}\left(D_{j}^{\top}, D_{p(-)}^{\top} ; \omega\right) \\
& =Q_{(-)}^{0}\left(W^{j}, W^{p} ; \Xi\right)+\left(A_{(-)}^{0}\right)_{j p} \text { mes }_{3} \omega,
\end{aligned}
$$


where mes $_{3} \omega$ is the volume of $\omega$. Note that, first, the equality $N^{0} W^{j}=-N^{0} D_{j}^{\top}$ on $\partial \omega$ is inherited from (3.35) and (3.15), second, $n^{\omega}$ and $-n^{\omega}$ imply the outward normals with respect to the sets $\Xi$ and $\omega$, respectively, and, third,

$$
\begin{aligned}
Q^{0}\left(u, v_{(-)} ; \Xi\right) & =\left(A^{0} D\left(\nabla_{\xi}\right) u, D\left(\nabla_{\xi}\right) v_{(-)}\right)_{\Xi} \\
& =\left(A_{(-)}^{0} D\left(\nabla_{\xi}\right) u, D\left(\nabla_{\xi}\right) v\right)_{\Xi}=Q_{(-)}^{0}(u, v ; \Xi), \\
Q_{(-)}^{0}\left(D_{j}^{\top}, D_{p}^{\top} ; \omega\right) & =\left(A_{(-)}^{0} \mathbf{e}_{j}, \mathbf{e}_{p}\right)_{\omega}=\left(A_{(-)}^{0}\right)_{j p} m_{e s} \omega .
\end{aligned}
$$

Comparing (3.45) and (3.46), we send $R$ to $+\infty$ and obtain the desired relation (3.41)

Theorem 12 ensures the matrix $M_{(=)}$in (3.40) to be symmetric, in particular, $M^{\mathrm{ME}}=-\left(M^{\mathrm{EM}}\right)^{\top}$. However, in contrast to the polarization matrix in elasticity (cf. [53, 38, 44) neither $M_{(=)}$, nor $M$ enjoy the positivity/negativity property. In the case $A^{\mathrm{ME}}=\mathbb{O}_{6 \times 3}$ the piezoelectricity problem decouples into the elasticity and electricity problems so that,

$$
M^{\mathrm{MM}}<0, M^{\mathrm{EE}}>0, M^{\mathrm{ME}}=-\left(M^{\mathrm{EM}}\right)^{\top}=\mathbb{O}_{6 \times 3},
$$

provided, e.g., mes $_{3} \omega>0$. We emphasize that in (3.48) $M^{\mathrm{EE}}$ is but the virtual mass tensor (see [48]). By the perturbation argument, the matrix $M$ has six negative and three positive eigenvalues, if the matrix $A^{\mathrm{ME}}$ is sufficiently small (cf. Section 4.4). However, for arbitrary $A^{\mathrm{ME}}$, this property is still an open question.

We have examined the first asymptotic term (3.36) of the boundary layer type in the asymptotic ansatz (3.19). By the representation (3.38) (see Remark 10), we write the expansion of $w^{1}(\xi)$ for $\xi \rightarrow+\infty$ in the matrix form as follows

$$
w^{1}(\xi)=\left(D\left(\nabla_{x}\right) \Phi(\xi)^{\top}\right)^{\top} M^{\top} \varepsilon^{0}+\widetilde{w}^{1}(\xi) .
$$

The remainder $\widetilde{w}^{1}$ obeys the estimates (3.32).

Remark 13 Formula (3.49) can be derived in the following way:

$$
\begin{aligned}
W^{j}(\xi) & =\sum_{p=1}^{9} M_{j p} \sum_{q=1}^{3} \frac{\partial \Phi}{\partial \xi_{q}}(\xi) D_{p}\left(e_{q}\right)^{\top}+\widetilde{W}^{j}(\xi) \\
& =\sum_{p=1}^{9} M_{j p}\left(\sum_{q=1}^{3} D_{p}\left(e_{q}\right) \frac{\partial \Phi}{\partial \xi_{q}}(\xi)^{\top}\right)^{\top}+\widetilde{W}^{j}(\xi) \\
& =\left(\sum_{p=1}^{9} M_{j p} D_{p}\left(\nabla_{\xi}\right) \Phi(\xi)^{\top}\right)^{\top}+\widetilde{W}^{j}(\xi) .
\end{aligned}
$$

\subsection{The second term in the boundary layer}

The system of differential equations in $\Xi$ in the exterior problem (3.23) for the boundary layer $w^{1}$ is homogeneous. This leads to relatively simple formulae (3.36) and (3.49) for $w^{1}$. However, $w^{2}$ is determined from problem (3.24) which enjoys the inhomegeneities both, in the boundary conditions and in the differential equations. Hence, the immediate objective becomes an inspection of the right-hand side $-L^{\prime} w^{1}$ for possible compensation and furthermore, an application of the same procedure as it is described in Section 3.3. However, the resulting asymptotic form (3.59) of $w^{2}$ looks quite different compared to (3.49).

By virtue of (3.16) and (3.17), the operator

$$
L^{\prime}\left(\xi, \nabla_{\xi}\right)=D\left(-\nabla_{\xi}\right)^{\top}\left(\sum_{j=1}^{3} \xi_{j} A^{j} D\left(\nabla_{\xi}\right)\right)
$$


gets the following homogeneity property:

$$
L^{\prime}\left(\xi, \nabla_{\xi}\right) \rho^{\lambda} \varphi(\theta)=\rho^{\lambda-1} \psi(\theta), \xi \in \mathbb{R}^{3} \backslash\{0\} .
$$

Here $\lambda \in \mathbb{R},(\rho, \theta)$ are the spherical coordinates in $\mathbb{R}^{3}, \rho=|\xi|$ and $\theta=\rho^{-1} \xi \in \mathbb{S}_{1}$, and $\varphi, \psi \in C^{\infty}\left(\mathbb{S}_{1}\right)^{4}$. Thus, by means of (3.36) and (3.38), (3.32), (3.30), we obtain that

$$
F^{\prime}(\xi)=-L^{\prime}\left(\xi, \nabla_{\xi}\right) w^{1}(\xi)=D\left(\nabla_{\xi}\right)^{\top}\left(\rho^{-2} \Psi(\xi)\right)+O\left(\rho^{-2}\right), \rho \rightarrow+\infty,
$$

while the formula can be differentiated under the standard convention $\nabla_{x} O\left(\rho^{-\lambda}\right)=O\left(\rho^{-\lambda-1}\right)$. Due to the definition (3.28) of the Kondratiev norm the right-hand side of (3.52) gives rise to the continuous functional

$$
\begin{gathered}
V_{0}^{1}(\Xi)^{4} \ni v \mapsto\left(F^{\prime}, v\right)_{\Xi} \\
\left|\left(F^{\prime}, v\right)_{\Xi}\right| \leq c \int_{\Xi} \rho^{-3}|v(\xi)| d \xi \leq c\left(\int_{\Xi} \rho^{-4} d \xi\right)^{1 / 2}\left\|\rho^{-1} v ; L^{2}(\Xi)\right\| \leq C\left\|v ; V_{0}^{1}(\Xi)\right\| .
\end{gathered}
$$

Thus, similarly to Proposition[7 we obtain the existence of a unique solution $w^{2} \in V_{0}^{1}(\Xi)^{4}$ to the problem (3.24). Now, we need to examine the behavior of $w^{2}(\xi)$ as $\rho \rightarrow+\infty$. According to 20] (see also 39, $\S 3.5]$ ), first of all, we have to determine the power-law solution

$$
Z(\xi)=\rho^{-1} \mathcal{Z}(\theta)
$$

to the system of differential equations

$$
L^{0}\left(\nabla_{\xi}\right) Z(\xi)=\rho^{-3} \mathcal{F}(\theta):=D\left(\nabla_{x}\right)^{\top}\left(\rho^{-2} \Psi(\theta)\right), \xi \in \mathbb{R}^{3} \backslash\{0\},
$$

with the right-hand side taken from (3.52). Note that, in general, the multiplier $\mathcal{Z}$ in (3.53) may be linear in $\ln \rho$ but, owing to a special form of $\mathcal{T}$, the next lemma proves the absence of the logarithm.

Lemma 14 The system (3.54) admits the power-law solution of form (3.53), whose angular part $\mathcal{Z}(\theta)$ is defined up to the linear combination $c_{1} \Phi^{1}(\theta)+\cdots+c_{4} \Phi^{4}(\theta)$, where $c_{j} \in \mathbb{R}$ are arbitrary and $\Phi^{j}(\theta)$ is the trace on the unit sphere $\mathbb{S}_{1}$ of the column $\Phi^{j}(\xi)$ in the fundamental matrix $\Phi$.

Proof. After separation of variables and rewriting the operator $L^{0}\left(\nabla_{\xi}\right)=\rho^{-2} \mathfrak{L}\left(\theta, \nabla_{\theta}, \rho \partial_{\rho}\right)$ in the spherical coordinates $(\rho, \theta)$, the system (3.54) takes the form

$$
\mathfrak{L}\left(\theta, \nabla_{\theta},-1\right) \mathcal{Z}(\theta)=\mathcal{F}(\theta), \theta \in \mathbb{S}_{1} .
$$

By the Fredholm alternative, this system on the unit sphere has a solution if and only if the right-hand side $\mathcal{F}$ is orthogonal to all solutions of the formally adjoint homogeneous system. Owing to 29] (see also 39. Lemma 3.5.9]), the formally adjoint operator for $\mathfrak{L}\left(\theta, \nabla_{\theta},-1\right)$ is nothing but $\mathfrak{L}_{(\top)}\left(\theta, \nabla_{\theta}, 0\right)$, where

$$
\rho^{-2} \mathfrak{L}_{(\top)}\left(\theta, \nabla_{\theta}, \rho \partial_{\rho}\right)=L_{(\top)}^{0}\left(\nabla_{\xi}\right)=L^{0}\left(\nabla_{\xi}\right)^{*} .
$$

By virtue of the polynomial property (3.27), any power-law solution $X(\xi)=\rho^{0} \mathcal{X}(\xi)$ of $L_{(\top)}^{0}\left(\nabla_{\xi}\right) X=0$ in $\mathbb{R}^{3} \backslash\{0\}$ is a constant column in $\mathbb{R}^{4}$. Thus, it suffices to verify the orthogonality condition

$$
\int_{\mathbb{S}_{1}} \mathcal{F}(\theta) d s_{\theta}=0 \in \mathbb{R}^{4} .
$$

Let $R>r>0$ and let $\Theta$ be the annulus $\{\xi: r<\rho<R\}$. We have

$$
\begin{gathered}
\ln \left(\frac{R}{r}\right) \int_{\mathbb{S}_{1}} \mathcal{F}(\theta) d s_{\theta}=\int_{r}^{R} \rho^{-1} d \rho \int_{\mathbb{S}_{1}} \mathcal{F}(\theta) d s_{\theta}=\int_{\Theta} \rho^{-3} \mathcal{F}(\theta) d \xi=\int_{\Theta} D\left(\nabla_{\xi}\right)^{\top}\left(\rho^{-2} \psi(\theta)\right) d \xi \\
=\int_{\mathbb{S}_{R}} D\left(\rho^{-1} \xi\right)^{\top}\left(\rho^{-2} \Psi(\theta)\right) d s_{\xi}-\int_{\mathbb{S}_{r}} D\left(\rho^{-1} \xi\right)^{\top}\left(\rho^{-2} \Psi(\theta)\right) d s_{\xi}=0 .
\end{gathered}
$$


We have used here the Gauss formula and the fact that the integrands at $\rho=R$ and $\rho=r$ are equal to $R^{-2} D(\theta)^{\top} \Psi(\theta)$ and $r^{-2} D(\theta)^{\top} \Psi(\theta)$, respectively, so that the integrals cancel each other.

Thus, the compatibility condition (3.57) holds true and the system (3.55) admits a solution. It remains to recall that any power-law solution (3.53) of the homogeneous system (3.54) becomes a linear combination of the fundamental matrix columns.

To assure the uniqueness of the solution (3.53), we impose the condition

$$
\int_{\mathbb{S}_{1}} D(\theta)^{\top} A^{0} \mathfrak{D}\left(\theta, \nabla_{\theta},-1\right) \mathcal{Z}(\theta) d s_{\theta}=0 \in \mathbb{R}^{4}
$$

where $\rho^{-1} \mathfrak{D}\left(\theta, \nabla_{\theta}, \rho \partial_{\rho}\right)$ is the matrix operator $D\left(\nabla_{x}\right)$ written, similarly to (3.56), in the spherical coordinates $(\rho, \theta)$.

Now, we are in position to write an expansion at infinity for the second boundary layer term in (3.19).

Proposition 15 The solution $w^{2} \in V_{0}^{1}(\Xi)^{4}$ of the problem (3.24) admits the asymptotic form

$$
\begin{gathered}
w^{2}(\xi)=Z(\xi)+\Phi(\xi) C+\widetilde{w}^{2}(\xi), \\
\left|\nabla_{\xi}^{k} \widetilde{w}^{2}(\xi)\right| \leq c_{k, \beta} \rho^{-2-k+\beta}, k \in \mathbb{N}_{0}, \xi \in \mathbb{R}^{3} \backslash \mathcal{V},
\end{gathered}
$$

where $\beta>0$ is arbitrary, $Z$ is a power-law solution of form (3.53) and $C \in \mathbb{R}^{4}$ is determined as follows:

$$
\begin{array}{r}
C=-f(0) \text { mes }_{3} \omega+J \in \mathbb{R}^{4}, \\
J=\int_{\mathbb{S}_{1}} D(\theta)^{\top} \sum_{j=1}^{3} \xi_{j} A^{j} D\left(\nabla_{\xi}\right)\left(D\left(\nabla_{\xi}\right) \Phi(\xi)^{\top}\right)^{\top} d s_{\xi} M^{\top} \varepsilon^{0} .
\end{array}
$$

Proof. The asymptotic expansion (3.59) with a certain column $C$ and the estimates (3.60) result from [20 and [30, respectively (see also [39, Ch.3]). We again employ the method proposed in [29] to evaluate the constant column $C$. Now, we use the Green formula in $\Xi_{R}$ for $w^{2}$ and $\mathrm{e}_{\mathrm{p}}=\left(\delta_{\mathrm{p}, 1}, \ldots, \delta_{\mathrm{p}, 4}\right)^{\top}$. Recalling (3.24), we have

$$
\begin{aligned}
& I_{\text {left }}:=-\int_{\Xi_{R}} \mathrm{e}_{p}^{\top} L^{\prime} w^{1} d \xi-\int_{\partial \omega} \mathrm{e}_{p}^{\top} N^{\prime} w^{1} d s \xi-\int_{\partial \omega} \mathrm{e}_{p}^{\top} N^{\prime} D(\xi) \varepsilon^{0} d s_{\xi}-\int_{\partial \omega} \mathrm{e}_{p}^{\top} N^{\prime} U d s_{\xi} \\
& =\int_{\Xi_{R}} \mathrm{e}_{p}^{\top} L^{0} w^{2} d \xi+\int_{\partial \omega} \mathrm{e}_{p}^{\top} N^{0} w^{2} d s \xi=\int_{\mathbb{S}_{R}} \mathrm{e}_{p}^{\top} N^{0} w^{2} d s \xi=: I_{\text {right }} .
\end{aligned}
$$

Here $N^{0}\left(\xi, \nabla_{\xi}\right)=D(\theta)^{\top} A^{0} D\left(\nabla_{\xi}\right)$ on the sphere $\mathbb{S}_{R}$ with the unit normal vector $\theta=\rho^{-1} \xi$ (cf. (3.22) and (3.58)). Similarly to the calculation (3.45), using (3.59) and (3.58), we get

$$
\begin{aligned}
I_{\text {right }} & =-\int_{\mathbb{S}_{R}} \mathrm{e}_{p}^{\top} N^{0} \mathcal{Z} d s_{\xi}-\int_{\mathbb{S}_{R}} \mathrm{e}_{p}^{\top} N^{0} \Phi d s_{\xi} C+O\left(R^{-1}\right)= \\
& =\int_{\mathbb{B}_{R}} \mathrm{e}_{p}^{\top} L^{0} \Phi d \xi C+O\left(R^{-1}\right)=C_{p}+O\left(R^{-1}\right) .
\end{aligned}
$$

By integrating by parts, the last couple of integrals in $I_{l e f t}$ turns into

$$
-\int_{\Xi_{R}} \mathrm{e}_{p}^{\top} L^{\prime} w^{1} d \xi-\int_{\partial \omega} \mathrm{e}_{p}^{\top} N^{\prime} w^{1} d s \xi=\int_{\mathbb{S}_{R}} \mathrm{e}_{p}^{\top} D(\theta)^{\top} \sum_{j=1}^{3} \xi_{j} A^{j} D\left(\nabla_{\xi}\right)\left(D\left(\nabla_{\xi}\right) \Phi(\xi)^{\top}\right)^{\top} d s_{\xi} M \varepsilon^{0}+O\left(R^{-1}\right) .
$$

Here we have applied the decomposition (3.49) of $w^{1}$ together with the estimate (3.32) for the remainder. Since its integrand is a positive homogeneous function in $\xi$ of degree -2 (cf. (3.30) ) the integral $J_{p}$ over $\mathbb{S}_{R}$ in (3.65) is independent of the radius $R$ and becomes an entry of column (3.62). 
The first couple of integrals in (3.63) is equal to

$$
\begin{aligned}
& \int_{\partial \omega} \mathrm{e}_{p}^{\top} N^{\prime} D(\xi) \varepsilon^{0} d s_{\xi}-\int_{\partial \omega} \mathrm{e}_{p}^{\top} N^{0} U d s_{\xi}=-\int_{\omega} \mathrm{e}_{p}^{\top}\left(L^{\prime} D(\xi) \varepsilon^{0}+L^{0} U\right) d \xi \\
& =-m e s_{3} \omega \mathrm{e}_{p}^{\top}\left(-\sum_{j=1}^{3} D\left(e_{j}\right)^{\top} A^{j} \varepsilon^{0}+L^{0}\left(\nabla_{\xi}\right) U(\xi)\right)=-f_{p}(0) m e s_{3} \omega .
\end{aligned}
$$

Here, the elementary formula (3.18) has been taken into account.

Now the limit passage $R \rightarrow+\infty$ in (3.63)-(3.65) furnishes (3.61) and (3.62)

Remark 16 Proposition 11 can be proved by an application of the method [29] in the same way as it is made in Proposition 15 and Theorem [12. We only mention that the columns $d^{1}, \ldots, d^{7}$ of the matrix $d(\xi)$ in (3.13) satisfy simultaneously the homogeneous problem (3.23) and the formally adjoint boundary value problem in $\Xi$ with the operators $L_{(\top)}^{0}\left(\nabla_{\xi}\right)$ and $N_{(\top)}^{0}\left(\xi, \nabla_{\xi}\right)$, respectively.

\subsection{The regular correction term}

Let us consider now the subsequent term in the asymptotic ansatz (3.19), namely the regular correction term $\mathbf{u}(x)$.

By means of (3.49) and (3.59), we have

$$
\begin{aligned}
& h w^{1}\left(h^{-1} x\right)+h^{2} w^{2}\left(h^{-1} x\right)=h\left(S^{2}\left(h^{-1} x\right)+\widetilde{w}^{1}\left(h^{-1} x\right)\right)+ \\
& +h^{2}\left(S^{1}\left(h^{-1} x\right)+\widetilde{w}^{2}\left(h^{-1} x\right)\right)=h^{3}\left(S^{2}(x)+S^{1}(x)\right)+O\left(h^{4}\left(|x|^{-3}+|x|^{-2}\right)\right)
\end{aligned}
$$

where, according to (3.30) and (3.53), we have set

$$
\begin{gathered}
S^{2}(\xi)=\left(D\left(-\nabla_{\xi}\right) \Phi(\xi)^{\top}\right)^{\top} M^{\top} \varepsilon^{0}, S^{1}(\xi)=Z(\xi)+\Phi(\xi) C, \\
S^{p}(t \xi)=t^{-p} S^{p}(\xi) .
\end{gathered}
$$

Therefore, this is $h^{3} \mathbf{u}(x)$ in the asymptotic ansatz (3.19) that compensates the main part of a discrepancy produced by the boundary layer terms $w^{1}$ and $w^{2}$.

Taking into account the equalities $L^{0} S^{2}=0$ and $L^{0} S^{1}=-L^{\prime} S^{2}$ designated in two last sections, we arrive at the following representation of the discrepancy in the system (3.2) :

$$
\begin{aligned}
& \mathbf{f}(x)=-L\left(x, \nabla_{x}\right)\left(\chi(x)\left(S^{2}(x)+S^{1}(x)\right)\right)= \\
& =-[L, \chi]\left(S^{2}(x)+S^{1}(x)\right)-\chi(x)\left(L\left(x, \nabla_{x}\right)-L^{0}\left(\nabla_{x}\right)-L^{\prime}\left(x, \nabla_{x}\right)\right) S^{2}(x)- \\
& -\chi(x)\left(L\left(x, \nabla_{x}\right)-L^{0}\left(\nabla_{x}\right)\right) S^{1}(x) .
\end{aligned}
$$

Here $[L, \chi]$ stands for the commutator of the differential operator $L$ and the cut-of function $\chi$, i.e.,

$$
[L, \chi]=D\left(-\nabla_{x}\right)^{\top} A(x) D\left(\nabla_{x} \chi(x)\right)-D\left(\nabla_{x} \chi(x)\right)^{\top} A(x) D\left(\nabla_{x}\right) .
$$

Recalling (3.16) and (3.17), in view of (3.67), we obtain that

$$
|\mathbf{f}(x)| \leq c|x|^{-2} .
$$

We see that the regular correction term $\mathbf{u}$ must satisfy the piezoelectricity problem

$$
\begin{array}{r}
D\left(-\nabla_{x}\right)^{\top} A(x) D\left(\nabla_{x}\right) \mathbf{u}(x)=\mathbf{f}(x), x \in \Omega, \\
D(n(x))^{\top} A(x) D\left(\nabla_{x}\right) \mathbf{u}(x)=0, x \in \Gamma_{\sigma}, \mathbf{u}(x)=0, x \in \Gamma_{u} .
\end{array}
$$

We emphasize that the sum $h \widetilde{w}^{1}\left(h^{-1} x\right)+h^{2} \widetilde{w}^{2}\left(h^{-1} x\right)$ in (3.66) becomes of order $h^{4}$ only at a distance from the coordinate origin $x=0$. However, we have extended equations (3.71) over the whole domain $\Omega$ because the singularity $O\left(|x|^{-2}\right)$ of the right-hand side $\mathbf{f}(x)$ is not too strong. In particular, by (3.70), the functional on the right-hand side in the integral identity

$$
Q(\mathbf{u}, \mathbf{v} ; \Omega)=(\mathbf{f}, \mathbf{v})_{\Omega}, \mathbf{v} \in \stackrel{\circ}{H}^{1}\left(\Omega ; \Gamma_{u}\right)^{4},
$$


serving for the problem (3.71), (3.72) (cf. (2.12)), is continuous due to the estimate

$$
\begin{aligned}
& \left|(\mathbf{f}, \mathbf{v})_{\Omega}\right| \leq c\left(\int_{\Omega}|x|^{2}|\mathbf{f}(x)|^{2} d x\right)^{1 / 2}\left(\int_{\Omega}|x|^{-2}|\mathbf{v}(x)|^{2} d x\right)^{1 / 2} \leq \\
& \leq c\left(\int_{0}^{\operatorname{diam} \Omega} r^{2} r^{-4} r^{2} d r\right)^{1 / 2}\left\|\nabla_{x} \mathbf{v} ; L_{2}(\Omega)\right\| \leq C\left\|\mathbf{v} ; H^{1}(\Omega)\right\|
\end{aligned}
$$

and the one-dimensional Hardy inequality mentioned above (3.28). Hence, in the analogy with Proposition 1. the Lax-Milgram lemma ensures the existence and uniqueness of the solution $\mathbf{u} \in \stackrel{H}{ }^{1}\left(\Omega ; \Gamma_{u}\right)^{4}$. These observations complete the evaluation of all asymptotic terms detached in (3.19).

Remark $\mathbf{1 7}$ The singularity of $\mathbf{f}$ can lead to a logarithmical singularity of the solution $\mathbf{u}$. However, we shall need only the following inequalities with arbitrary $\beta>0$ :

$$
|\mathbf{u}(x)| \leq c_{\beta}|x|^{-\beta},\left|\nabla_{x} \mathbf{u}(x)\right| \leq c_{\beta}|x|^{-1-\beta}
$$

delivered by a result in [30] (see also [39, §3.6]).

For the further usage, it is convenient to rewrite the ansatz (3.19) in a different form, namely

$$
u^{h}(x)=u(x)+h^{3} \mathbf{U}(x)+\chi(x)\left(h \widetilde{w}^{1}\left(h^{-1} x\right)+h^{2} \widetilde{w}^{2}\left(h^{-1} x\right)\right)+\widetilde{u}^{h}(x),
$$

where, in accordance with (3.66) and (3.67),

$$
\mathbf{U}(x)=\mathbf{u}(x)+\chi(x)\left(S^{2}(x)+S^{1}(x)\right) .
$$

In other words, we detach $h S^{2}\left(h^{-1} x\right)$ and $h^{2} S^{1}\left(h^{-1} x\right)$ from the boundary layer terms and attach them to the regular term $\mathbf{u}$. Therefore, the remainder $\widetilde{u}^{h}$ in (3.75) stays the same as in the original ansatz (3.19).

Let us derive an almost explicit formula for (3.76). To this end, let $G(x, y)$ be the Green matrix for the piezoelectricity problem (2.9)-(2.11), i.e.,

$$
\begin{aligned}
& D\left(-\nabla_{x}\right)^{\top} A(x) D\left(\nabla_{x}\right) G(x, y)=\delta(x-y) \mathbb{I}_{4 \times 4}, x \in \Omega, \\
& D(n(x))^{\top} A(x) D\left(\nabla_{x}\right) G(x, y)=0, x \in \Gamma_{\sigma}, u(x)=0, x \in \Gamma_{u}
\end{aligned}
$$

Of course, the relations (3.77) are understood in the sense of distributions, so that, $G \in L^{2}(\Omega)^{4 \times 4}$, $G \in L^{2}(\partial \Omega)^{4 \times 4}$ and

$$
\left(G, L_{(\top)} v\right)_{\Omega}+\left(G, N_{(\top)} v\right)_{\Gamma_{\sigma}}=v(y), v \in C_{c}^{\infty}\left(\bar{\Omega} ; \Gamma_{u}\right)^{4},
$$

where the linear space $C_{0}^{\infty}\left(\bar{\Omega} ; \Gamma_{u}\right)$ consists of infinitely differentiable functions in $\bar{\Omega}$ which vanish on $\Gamma_{u}$. Since $A$ is a smooth matrix function inside of the ball $\mathbb{B}_{R}$ (see (3.7)), the Green matrix is properly defined for $y \in \mathbb{B}_{R}$ (see [11, 13]) and

$$
(x \mapsto G(x, y)-\Phi(x, y)) \in H^{1}(\Omega)^{4 \times 4} .
$$

Moreover, $G$ can be differentiated in the second argument and we set

$$
G^{0}(x)=G(x, 0), \mathbf{G}^{0}(x)=\left.D\left(-\nabla_{y}\right) G(x, y)\right|_{y=0} .
$$

By repeating the considerations in and around of Lemma 14 we can detect that

$$
G^{0}-\Phi \in H^{1}(\Omega)^{4 \times 4}, \mathbf{G}^{0}-D\left(\nabla_{x}\right) \Phi-\mathbf{Z}-\mathbf{K} \Phi \in H^{1}(\Omega)^{9 \times 4},
$$

where $\mathbf{K}$ is a certain matrix of the size $9 \times 4$ with real entries and $\mathbf{Z}$ is such that $Z(x)=\mathbf{Z}(x) M^{\top} \varepsilon^{0}$ (cf. (3.50) and (3.52)-(3.54)). Since, by definition of $\mathbf{u}$ and $S^{q}$, the vector function $\mathbf{U}$ verifies the boundary conditions (3.72) and the homogeneous system (3.71) everywhere in $\Omega$, except at the point $\mathcal{O}$. Let us now compare singularities in (3.79) and (3.76) to conclude that

$$
\mathbf{U}(x)=\mathbf{G}^{0}(x) M^{\top} \varepsilon^{0}-G^{0}(x) f(0) \text { mes }_{3} \omega .
$$


Remark 18 1. We emphasize that the differential operator $D\left(-\nabla_{y}\right)$ in (3.78) is replaced by $D\left(\nabla_{x}\right)$ in (3.79). This is due to the evident relationship $D\left(-\nabla_{y}\right) \Phi(x-y)=D\left(\nabla_{x}\right) \Phi(x-y)$.

2. If $A$ is a constant matrix, then the terms $\mathbf{Z}$ and $\mathbf{K} \Phi$ are absent in (3.79), in other words, their presence results from the variable coefficients of differential operator (3.17). Therefore, the column $-f(0) m^{2} s_{3} \omega$ occurs on the right-hand side of (3.80) instead of the column (3.61). To ensure that the additional column (3.62) does not effect the form of the last term in (3.80), one may put $\varepsilon^{0}=0$ to see that then $J=0$. A direct calculation leading to formula (3.80) can be found in [43] for the three-dimensional elasticity problem.

Since the coordinate origin $\mathcal{O}$ is situated inside $\omega_{h}$, i.e., outside $\bar{\Omega}_{h}$ (cf. Section 3.1), the second term (3.80) in the new ansatz (3.75) is smooth in the domain $\Omega(h)$, although the Green matrices (3.78) have singularities at $\mathcal{O}$.

\section{Justification of asymptotics and analysis of shape functionals}

\subsection{The justification of asymptotics}

The difference

$$
\widetilde{u}^{h}=u^{h}-u-\chi\left(h w^{1}+h^{2} w^{2}\right)-h^{3} \mathbf{u}
$$

(see (3.19) and (3.75) ) satisfies the integral identity

$$
Q\left(\widetilde{u}^{h}, v ; \Omega(h)\right)=\widetilde{\mathcal{F}}^{h}(v), v \in \stackrel{\circ}{H}^{1}\left(\Omega(h) ; \Gamma_{u}\right)^{4},
$$

where $\widetilde{\mathcal{F}}^{h}$ is a certain functional (see, e.g., 4.5) ). If the estimate

$$
\left|\widetilde{\mathcal{F}}^{h} v\right| \leq c h^{\alpha+5 / 2}\left\|v ; H^{1}(\Omega(h))\right\|
$$

is proved, we could take $v=\widetilde{u}^{h}$ in order to conclude by using (2.14) that

$$
\left\|\widetilde{u}^{h} ; H^{1}(\Omega(h))\right\| \leq c h^{\alpha+5 / 2} .
$$

In the sequel, it is shown, that the constants in (4.3) and (4.4) are independent of the small parameter $h$.

To verify (4.3), first, we assume that $v$ vanishes in the ball $\mathbb{B}_{2 R / 3}$, therefore, $\chi v=0$. Then, we have

$$
\widetilde{\mathcal{F}}^{h}(v)=Q\left(u^{h}-u-h^{3} \mathbf{u}, v ; \Omega(h)\right)=Q\left(u^{h}, v ; \Omega(h)\right)-Q(u, v ; \Omega)-h^{3} Q(\mathbf{u}, v ; \Omega) .
$$

Recalling (3.6), (2.12) and (3.73), we observe that the support of the vector function (3.68) satisfies supp $\mathbf{f} \subset \overline{\mathbb{B}}_{2 R / 3}$ (each term in (3.68) contains either a cut-off function $\chi$ supported in the ball, or its derivatives) and, hence, 4.5 is null.

Second, let supp $v \subset \mathbb{B}_{R} \backslash \omega_{h}$. We write

$$
\begin{aligned}
& \widetilde{F}^{h}(v)=(f, v)_{\Omega(h)}-\left(A D\left(\nabla_{x}\right) u, D\left(\nabla_{x}\right) v\right)_{\Omega(h)}-h^{3}\left(A D\left(\nabla_{x}\right) \mathbf{u}, D\left(\nabla_{x}\right) v\right)_{\Omega(h)} \\
& -h\left(A D\left(\nabla_{x}\right)\left(\chi w^{1}\right), D\left(\nabla_{x}\right) v\right)_{\Omega(h)}-h^{2}\left(A D\left(\nabla_{x}\right)\left(\chi w^{2}\right), D\left(\nabla_{x}\right) v\right)_{\Omega(h)} \\
& =:(f, v)_{\Omega(h)}-I^{u}-h^{3} I^{\mathbf{u}}-h I_{1}^{w}-h^{2} I_{2}^{w} .
\end{aligned}
$$


Since the vector functions $u$ and $\mathbf{u}$ are smooth in $\mathbb{B}_{R} \backslash \omega_{h}$, we integrate by parts and obtain

$$
\begin{array}{r}
I^{u}=(f, v)_{\Omega(h)}+\left(D\left(n^{h}\right)^{\top} A^{0} \varepsilon^{0}, v\right)_{\partial \omega_{h}}+\left(D\left(n^{h}\right)^{\top} A^{0} D\left(\nabla_{x}\right) U, v\right)_{\partial \omega_{h}} \\
+\sum_{j=1}^{3}\left(D\left(n^{h}\right)^{\top} x_{j} A^{j} \varepsilon^{0}, v\right)_{\partial \omega_{h}}+\widetilde{I}^{u}, \\
\widetilde{I}^{u}=\left(D\left(n^{h}\right)^{\top} A D\left(\nabla_{x}\right)\left(u-D(x)^{\top} \varepsilon^{0}-U\right), v\right)_{\partial \omega_{h}}+ \\
\left(D\left(n^{h}\right)^{\top}\left(A-A^{0}-\sum_{j=1}^{3} x_{j} A^{j}\right) \varepsilon^{0}, v\right)_{\partial \omega_{h}}+\left(D\left(n^{h}\right)^{\top}\left(A-A^{0}\right) D\left(\nabla_{x}\right) U, v\right)_{\partial \omega_{h}}, \\
I^{\mathbf{u}}=(\mathbf{f}, v)_{\Omega(h)}+\widetilde{I}^{\mathbf{u}}, \quad \widetilde{I}^{\mathbf{u}}=\left(D\left(n^{h}\right)^{\top} A D\left(\nabla_{x}\right) \mathbf{u}, v\right)_{\partial \omega_{h}} .
\end{array}
$$

To process the terms $\widetilde{I}^{u}$ and $\widetilde{I}^{\mathbf{u}}$, we recall the inequality

$$
\int_{\Omega(h)}|x|^{-2}|v(x)|^{2} d x \leq c\left\|v ; H^{1}(\Omega(h))\right\|^{2},
$$

which is a consequence of the one-dimensional Hardy inequality (cf. [39, §4.5]) and the trace inequality (see [23])

$$
\int_{\partial \omega_{h}}|v(x)|^{2} d s_{x} \leq c h\left\|v ; H^{1}(\Omega(h))\right\|^{2},
$$

where the constants $c$ are independent of $h \in\left(0, h_{0}\right]$ and $v$.

Now by (4.10) and (3.74), we readily derive that

$$
\begin{aligned}
h^{3}\left|\widetilde{I}^{\mathbf{u}}\right| \leq c h^{3} h^{-1-\beta} \int_{\partial \omega_{h}}|v(x)| d s_{x} & \leq c h^{2-\beta}\left(\text { mes }_{2} \partial \omega_{h}\right)^{1 / 2} h^{1 / 2}\left\|v ; H^{1}(\Omega(h))\right\| \\
& =C h^{-\beta+7 / 2}\left\|v ; H^{1}(\Omega(h))\right\| .
\end{aligned}
$$

Analogously, by means of (3.16), (3.11) and (4.10), we have

$$
\left|\widetilde{I}^{u}\right| \leq c\left(h^{1+\alpha}+h^{2}+h^{2}\right) \int_{\partial \omega_{h}}|v(x)| d s_{x} \leq c h^{\alpha+5 / 2}\left\|v ; H^{1}(\Omega(h))\right\| .
$$

We may choose $\beta=1-\alpha>0$ in order to equalize the final exponents of $h$ in (4.11) and (4.12).

Dealing with $I_{2}^{w}$, we write

$$
\begin{gathered}
I_{2}^{w}=\left(A D\left(\nabla_{x} \chi\right) S^{1}, D\left(\nabla_{x}\right) v\right)_{\Omega(h)}-\left(A D\left(\nabla_{x}\right) S^{1}, D\left(\nabla_{x} \chi\right) v\right)_{\Omega(h)} \\
+\left(A^{0} D\left(\nabla_{x}\right) w^{2}, D\left(\nabla_{x}\right)(\chi v)\right)_{\Omega(h)}+\widetilde{I}_{2}^{w}, \\
\widetilde{I}_{2}^{w}=\left(A D\left(\nabla_{x} \chi\right)\left(w^{2}-S^{1}\right), D\left(\nabla_{x}\right) v\right)_{\Omega(h)}-\left(A D\left(\nabla_{x} \chi\right)\left(w^{2}-S^{1}\right), D\left(\nabla_{x} \chi\right) v\right)_{\Omega(h)} \\
+\left(\left(A-A^{0}\right) D\left(\nabla_{x}\right) w^{2}, D\left(\nabla_{x}\right)(\chi v)\right)_{\Omega(h)} .
\end{gathered}
$$

Here, we detach $S^{1}\left(h^{-1} x\right)$ from $w^{2}\left(h^{-1} x\right)$ (cf. (3.66) $)$ and commute twice the differential operator $D\left(\nabla_{x}\right)$ with the cut-off function $\chi$ (see (3.69)).

In view of (3.16) and (3.59), the absolute value of the last expression in (4.14), multiplied by $h^{2}$ according to the definition of $I_{2}^{w}$ in (4.6), does not exceed the sum of the following two expressions:

$$
\begin{aligned}
& c h^{2} \int_{\Omega \backslash \mathbb{B}_{R} h}|x| h^{-1}\left(\frac{|x|}{h}\right)^{-3+\beta}\left|D\left(\nabla_{x}\right)(\chi(x) v(x))\right| d x \leq \\
& \leq \operatorname{ch} h^{4-\beta}\left(\int_{R h}^{\operatorname{diam} \Omega} r^{2} r^{-6+2 \beta} r^{2} d r\right)^{1 / 2}\left\|v ; H^{1}(\Omega(h))\right\| \leq \operatorname{ch}^{7 / 2}\left\|v ; H^{1}(\Omega(h))\right\|
\end{aligned}
$$


and

$$
\begin{aligned}
& \operatorname{ch}^{2} \int_{\mathbb{B}_{R h} \backslash \omega_{h}}|x|\left|D\left(\nabla_{x}\right) \widetilde{w}^{2}\left(h^{-1} x\right) \| D\left(\nabla_{x}\right)(\chi v)\right| d x \\
& \leq \operatorname{ch} h^{2} R h\left(\int_{\mathbb{B}_{R} \backslash \omega} h^{-2}\left|D\left(\nabla_{\xi}\right) \widetilde{w}^{2}(\xi)\right|^{2} d \xi h^{3}\right)^{1 / 2}\left\|v ; H^{1}(\Omega(h))\right\| \\
& \leq \operatorname{ch}{ }^{7 / 2}\left\|v ; H^{1}(\Omega(h))\right\| .
\end{aligned}
$$

The radius $R$ is chosen such that $\mathbb{B}_{R} \supset \bar{\omega}$. Since the support of $\left|D\left(\nabla_{x} \chi\right)\right|$ belongs to the annulus $\overline{\mathbb{B}_{2 R / 3}} \backslash \mathbb{B}_{R / 3}$ where, according to (3.59),

$$
\left|w^{2}\left(h^{-1} x\right)-S^{1}\left(h^{-1} x\right)\right|+\left|\nabla_{x}\left(w^{2}\left(h^{-1} x\right)-S^{1}\left(h^{-1} x\right)\right)\right| \leq c h^{2-\beta},
$$

the remaining terms in (4.14), again after multiplication by $h^{2}$, are bounded by $c h^{4-\beta}\left\|v ; H^{1}(\Omega)\right\|$ while we may set $\beta=1 / 2$ to achieve the same exponent as in (4.15). In other words, for $p=2$, we now have

$$
h^{p}\left|\widetilde{I}_{p}^{w}\right| \leq c h^{7 / 2}\left\|v ; H^{1}(\Omega(h))\right\| .
$$

By formulae (3.49), (3.32) and (3.66), the similar argument leads to the estimate (4.17) for the remainder in the representation

$$
\begin{aligned}
& I_{1}^{w}=\left(A D\left(\nabla_{x} \chi\right) S^{2}, D\left(\nabla_{x}\right) v\right)_{\Omega(h)}-\left(A D\left(\nabla_{x}\right) S^{2}, D\left(\nabla_{x} \chi\right) v\right)_{\Omega(h)}+ \\
& \left(A^{0} D\left(\nabla_{x}\right) w^{1}, D\left(\nabla_{x}\right)(\chi v)\right)_{\Omega(h)}+\sum_{j=1}^{3}\left(x_{j} A^{j} D\left(\nabla_{x}\right) w^{1}, D\left(\nabla_{x}\right)(\chi v)\right)_{\Omega(h)}+\widetilde{I}_{1}^{w} .
\end{aligned}
$$

Now, we are in position to conclude the estimate (4.3) for the functional $\widetilde{F}^{h}$ in (4.2), (4.5) and (4.6). To this end, we list several facts. First, the inner product $(f, v)_{\Omega(h)}$ on the right hand-side of (4.6) cancels the same product in (4.7). Second, the equality

$$
(\mathbf{f}, \mathbf{v})_{\Omega(h)}=-\left(A D\left(\nabla_{x} \chi\right)\left(S^{2}-S^{1}\right), D\left(\nabla_{x}\right) v\right)_{\Omega(h)}+\left(A D\left(\nabla_{x}\right)\left(S^{2}-S^{1}\right), D\left(\nabla_{x} \chi\right) v\right)_{\Omega(h)}
$$

is inherited from the definitions (3.68) and (3.69). Third, we make the coordinate dilation $x \mapsto \xi=h^{-1} x$ in the first couples of terms on the right hand-side of (4.13) and (4.18), simultaneously multiplying the terms by $h^{2}$ and $h$, respectively. Noting that $S^{p}\left(h^{-1} x\right)=h^{p} S^{p}(x), p=1,2$, we see that these couples and $h^{3}(\mathbf{f}, \mathbf{v})_{\Omega(h)}$ annihilate. Finally, we recall the integral identities (3.29), serving for the problems (3.23) and (3.24), and after the substitutions $x \mapsto \xi$ and $v(\xi) \mapsto \chi(h \xi) v(h \xi)$, we detect all terms in the identities on the right hand-sides of (4.7), (4.13) and (4.18). Thus,

$$
\widetilde{F}^{h}(v)=\widetilde{I}^{u}+h^{3} \widetilde{I}^{\mathbf{u}}+h \widetilde{I}_{1}^{w}+h^{2} \widetilde{I}_{2}^{w}
$$

and the inequality (4.3) holds true by virtue of (4.12), (4.11) and (4.17) with $p=1,2$. We notice that the lowest exponent $\alpha+5 / 2$ of $h$ occurs in (4.12) because $\alpha \in(1 / 2,1)$ and $\alpha+5 / 2 \in(3,7 / 2)$.

We now formulate the result.

Theorem 19 Let all assumptions in Section 3.1 be valid, in particular, the inclusion (3.9) with $\alpha \in$ $(1 / 2,1)$. Then the solution $u^{h}$ of the piezoelectricity problem (3.2)-(3.5) and its approximation constructed in Section $\mathbf{3}$ are in the relationship

$$
\left\|u^{h}-u-h^{3} \mathbf{u}-\chi\left(h w^{1}+h^{2} w^{2}\right) ; H^{1}(\Omega(h))\right\| \leq c h^{\alpha+5 / 2} N,
$$

where the constant $c$ is independent of the parameter $h \in\left(0, h_{0}\right]$ and the right-hand sides $f, g$ while

$$
N=\left\|f ; L^{2}(\Omega)\right\|+\left\|g ; L^{2}(\partial \Omega)\right\|+\left\|f ; C^{2, \alpha}\left(\mathbb{B}_{R}\right)\right\| .
$$

In asymptotic estimate (4.19) u stands for a solution of piezoelectricity mixed boundary value problem (2.9)-(2.11) in the intact body $\Omega$ and $\mathbf{u}$ for the main regular corrector which is a solution of problem 
(3.71), (3.72) in $\Omega$ and admits the representation (3.76) with the smooth addendum $\mathbf{U}$ and the singular components (3.67). The boundary layer terms $w^{1}$ and $w^{2}$, which are as well present in (4.19), are given by solutions to the exterior problems (3.23) and (3.24), decay at infinity and take asymptotic forms (3.49) and (3.59), respectively.

Remark 20 The obtained estimate (4.19) is asymptotically sharp, in particular, it satisfies the "first omitted term" rule. Indeed, for the smooth data $A$ and $f$, the subsequent asymptotic term in the ansatz (3.19) is $h^{3} \chi(x) w^{3}\left(h^{-1} x\right)$, the $H^{1}(\Omega(h))$-norm of the latter term is just $O\left(h^{7 / 2}\right)$. This bound appears in (4.20) if $\alpha \rightarrow 1-0$. Moreover, the estimate (4.19) holds true when the last addendum in (4.20) is changed for $\left\|f ; C^{3, \alpha_{1}}\left(\mathbb{B}_{R}\right)\right\|$ with any $\alpha_{1} \in(0,1)$. If the right-hand side $f \in C^{2, \alpha}\left(\mathbb{B}_{R}\right)^{4}$ in the equations (3.2) is not sufficiently smooth, e.g.,

$$
f(x)=f^{0}(x)+|x|^{2+\alpha} f^{1}(\theta), f^{0} \in C^{\infty}\left(\mathbb{B}_{R}\right)^{4}, f^{1} \in C^{\infty}\left(\mathbb{S}_{1}\right)^{4},
$$

then the asymptotic ansatz (3.19) gains the boundary layer term $h^{2+\alpha} \chi(x) w^{2+\alpha}\left(h^{-1} x\right)$ with the Sobolev norm in $\Omega(h)$ of the some order $h^{\alpha+5 / 2}$ as on the right hand-side of (4.19).

A direct calculation show that

$$
h^{j}\left\|\chi w^{j} ; H^{1}(\Omega(h))\right\|=O\left(h^{j+1 / 2}\right), j=1,2,
$$

and, therefore, in view of the relation $\alpha+5 / 2>3$ (see (3.9)), the $H^{1}(\Omega(h)$ )-norm of each of the detached asymptotic terms in (4.19) (cf. (3.19) and (3.75)) is of order $h^{s}$ with $s \leq 3$. In other words, Theorem 19 justifies the constructed asymptotics of solution $u^{h}$, indeed.

\subsection{The energy and the electric enthalpy}

We proceed with energy functional (2.17), assuming for simplicity that the volume forces and the volume charges are absent, i.e., $f=0$ on the right hand-sides of (2.9) and (3.2). Then, integrating by parts and taking into account formulae (3.75) and (4.4), we have

$$
\begin{aligned}
\mathcal{U}\left(u^{h} ; \Omega(h)\right) & =\frac{1}{2}\left(D(n)^{\top} A D\left(\nabla_{x}\right) u^{h}, u^{h}\right)_{\Gamma_{\sigma}}-\left(g, u^{h}\right)_{\Gamma_{\sigma}}=-\frac{1}{2}\left(g, u^{h}\right)_{\Gamma_{\sigma}} \\
& =-\frac{1}{2}(g, u)_{\Gamma_{\sigma}}-\frac{1}{2} h^{3}(g, \mathbf{u})_{\Gamma_{\sigma}}+O\left(h^{\alpha+5 / 2}\right) .
\end{aligned}
$$

Let $\mathfrak{v}^{\mathrm{M}} \in \stackrel{\circ}{H}^{1}\left(\Omega ; \Gamma_{u}\right)^{4}$ and $\mathfrak{v}^{\mathrm{E}} \in \stackrel{\circ}{H}^{1}\left(\Omega ; \Gamma_{u}\right)^{4}$ imply the solutions of the problem (2.9)-(2.11) with the right-hand sides

$$
\mathfrak{g}^{\mathrm{M}}=\left(g_{1}^{\mathrm{M}}, g_{2}^{\mathrm{M}}, g_{2}^{\mathrm{M}}, 0\right)^{\top}, \mathfrak{g}^{\mathrm{E}}=\left(0,0,0, g_{4}^{\mathrm{E}}\right)^{\top} .
$$

Using the representation (3.80) with $f(0)=0$ and the modified column $\mathbf{U}_{(-)}$(see (3.42)), we obtain

$$
\begin{aligned}
\left(\mathfrak{g}^{\mathrm{M}}, \mathbf{U}\right)_{\Gamma_{\sigma}} & =\left(\mathfrak{g}^{\mathrm{M}}, \mathbf{U}_{(-)}\right)_{\Gamma_{\sigma}}=\left(D(n)^{\top} A D\left(\nabla_{x}\right) \mathfrak{v}^{\mathrm{M}}, \mathbf{U}_{(-)}\right)_{\Gamma_{\sigma}} \\
& =\left(\mathfrak{v}^{\mathrm{M}}, D\left(-\nabla_{x}\right)^{\top} A^{\top} D\left(\nabla_{x}\right) \mathbf{U}_{(-)}\right)_{\Gamma_{\sigma}} \\
& =\left(\varepsilon^{0}\right)^{\top} M\left(\mathfrak{v}^{\mathrm{M}}, D\left(-\nabla_{x}\right)^{\top} A^{\top} D\left(\nabla_{x}\right) \mathbf{G}_{(-)}^{0}\right)_{\Omega} \\
& =\left(\varepsilon^{0}\right)^{\top} M\left(\mathfrak{v}^{\mathrm{M}},\left(D\left(\nabla_{x}\right)^{\top} \delta(x)\right)_{(-)}\right)_{\Omega}=-\left(\varepsilon^{0}\right)^{\top} M \mathfrak{e}_{(-)}^{\mathrm{M}}, \\
\left(\mathfrak{g}^{\mathrm{E}}, \mathbf{U}\right)_{\Gamma_{\sigma}} & =\left(\mathfrak{g}^{\mathrm{E}}, \mathbf{U}_{(-)}\right)_{\Gamma_{\sigma}}=-\left(\mathfrak{v}^{\mathrm{E}}, D\left(-\nabla_{x}\right)^{\top} A^{\top} D\left(\nabla_{x}\right) \mathbf{U}_{(-)}\right)_{\Gamma_{\sigma}} \\
& =\left(\varepsilon^{0}\right)^{\top} M \mathfrak{e}_{(-)}^{\mathrm{E}},
\end{aligned}
$$

where $\mathfrak{e}^{\mathrm{M}}=D\left(\nabla_{x}\right) \mathfrak{v}^{\mathrm{M}}(0)$ and $\mathfrak{e}^{\mathrm{E}}=D\left(\nabla_{x}\right) \mathfrak{v}^{\mathrm{E}}(0)$. Here, we apply formula (3.77) for the derivatives $\mathbf{G}^{0}$ of the Green matrix $G$ in (3.77). We emphasize that

$$
\left(f, \mathbf{G}_{(-)}^{0}\right)_{\Omega}+\left(g, \mathbf{G}_{(-)}^{0}\right)_{\Gamma_{\sigma}}=-\left(D\left(\nabla_{x}\right) u\right)_{(-)}(0)=-\varepsilon_{(-)}^{0}
$$

because entries of $\mathbf{G}^{0}$ are given by the derivatives of columns of the fundamental matrix $G(x, y)$ with respect to the second argument, and $G_{(-)}$satisfies the problem

$$
\begin{aligned}
& D\left(-\nabla_{x}\right)^{\top} A(x)^{\top} D\left(\nabla_{x}\right) G_{(-)}(x, y)=\delta(x-y) \mathbb{I}_{(-)}, x \in \Omega, \\
& D(n(x))^{\top} A(x)^{\top} D\left(\nabla_{x}\right) G_{(-)}(x, y)=0, x \in \Gamma_{\sigma}, G_{(-)}(x, y)=0, x \in \Gamma_{u},
\end{aligned}
$$


where $\mathbb{I}_{(-)}=\operatorname{diag}\{1,1,1,-1\}$ (cf. problem (3.77)).

By (4.22) and (4.24), the following representation is valid:

$$
\mathcal{U}\left(u^{h} ; \Omega(h)\right)-\mathcal{U}(u ; \Omega)=\frac{h^{3}}{2}\left(\varepsilon^{0}\right)^{\top} M\left(\mathfrak{e}_{(-)}^{\mathrm{M}}-\mathfrak{e}_{(-)}^{\mathrm{E}}\right)+O\left(h^{\alpha+5 / 2}\right) .
$$

At the first sight, (4.26) looks like (2.1), however this impression is wrong.

Remark 21 The decomposition $u=\mathfrak{v}^{\mathrm{M}}+\mathfrak{v}^{\mathrm{E}}$ is only a mathematical device in our analysis, since in a smart material it is impossible to distinguish between the strain columns $\mathfrak{e}^{\mathrm{M}}=D\left(\nabla_{x}\right) \mathfrak{v}^{\mathrm{M}}(0)$ and $\mathfrak{e}^{\mathrm{E}}=D\left(\nabla_{x}\right) \mathfrak{v}^{\mathrm{E}}(0)$ generated at the point $\mathcal{O}$ by the external mechanical loading $\mathfrak{g}^{\mathrm{M}}$ and the electrical surface charge $\mathfrak{g}^{\mathrm{E}}$ in (4.23). Surely, one can measure only the sum $\varepsilon^{0}=D\left(\nabla_{x}\right) u(0)$ resulting from complete external action and standing as the first term on the right hand-side of (4.26). This unususal property of the energy functional should always be taken into account and ignoring the above observation on the decomposition $u=\mathfrak{v}^{\mathrm{M}}+\mathfrak{v}^{\mathrm{E}}$ may provoke for misleading physical conclusions. Similar situation occurs for example for a crack in a piezoelectric medium. Applying the Griffith energy fracture criterion, in [46] the energy release rate at the crack tip is expressed in terms of stress intensity factors, i.e., local characteristics of the elastic/electric state at the tip. In [22] a mistake in a calculation (formulas (33.23) and (34.48) in [46, pages 296 and 312]; cf. comments in [22]) was discovered and a non-local formula for the energy release rate of type (4.26) was derived rigourously and justified. The non-local character of the energy release rate means that the energy functional $\mathcal{U}(u ; \Omega)$ cannot be employed for a fracture criterion and in the Griffith criterion must involve the electric enthalpy (cf. [15, 46] for an interpretation from the view point of solid fracture mechanics).

The difference

$$
\mathfrak{e}^{\mathrm{M}}-\mathfrak{e}^{\mathrm{E}}=\left(\mathfrak{g}^{\mathrm{E}}, \mathbf{G}^{0}\right)_{\Gamma_{\sigma}}-\left(\mathfrak{g}^{\mathrm{M}}, \mathbf{G}^{0}\right)_{\Gamma_{\sigma}}
$$

ought to be regarded as a global characteristics of the mechanical electric state of the body $\Omega$ and, therefore, formula (4.26) has a different physical meaning compared to (2.1) and (4.33) below.

Let us now compute the increment $\mathcal{E}\left(u^{h} ; \Omega(h)\right)-\mathcal{E}(u ; \Omega)$ of the mechanical enthalpy determined in (2.20) and (2.21). Returning back to the general case $f \neq 0$, we obtain

$$
\begin{aligned}
& \mathcal{E}\left(u^{h} ; \Omega(h)\right)=\frac{1}{2}\left(A D\left(\nabla_{x}\right) u^{h}, D\left(\nabla_{x}\right) u_{(-)}^{h}\right)_{\Omega(h)}-\left(f, u_{(-)}^{h}\right)_{\Omega}-\left(g, u_{(-)}^{h}\right)_{\Omega} \\
& =\frac{1}{2}\left(D\left(-\nabla_{x}\right)^{\top} A D\left(\nabla_{x}\right) u^{h}, u_{(-)}^{h}\right)_{\Omega(h)}+\frac{1}{2}\left(D(n)^{\top} A D\left(\nabla_{x}\right) u^{h}, u_{(-)}^{h}\right)_{\Gamma_{\sigma}} \\
& -\left(f, u_{(-)}^{h}\right)_{\Omega}-\left(g, u_{(-)}^{h}\right)_{\Omega}=-\frac{1}{2}\left(f, u_{(-)}^{h}\right)_{\Omega}-\frac{1}{2}\left(g, u_{(-)}^{h}\right)_{\Gamma_{\sigma}} .
\end{aligned}
$$

As above, we have

$$
\left(g, u_{(-)}^{h}\right)_{\Gamma_{\sigma}}=\left(g, u_{(-)}\right)_{\Gamma_{\sigma}}+h^{3}\left(g, \mathbf{U}_{(-)}\right)_{\Gamma_{\sigma}}+O\left(h^{\alpha+5 / 2}\right) .
$$

Furthermore, in view of representation (3.75) we derive

$$
\left(f, u_{(-)}^{h}\right)_{\Omega}=\left(f, u_{(-)}\right)_{\Omega}+h^{3}\left(f, \mathbf{U}_{(-)}\right)_{\Omega}+O\left(h^{\alpha+5 / 2}\right) .
$$

according to inequality (4.4) and the following relations

$$
\begin{array}{r}
h^{3}\left|\left(f, \mathbf{U}_{-}\right)_{\omega_{h}}\right| \leq c h^{3} \int_{\omega_{h}}|x|^{-2} d x \leq c h^{4} \leq c h^{\alpha+5 / 2}, \\
h\left|\left(f, \chi \widetilde{w}^{1}\right)_{\Omega(h)}\right| \leq c h \int_{0}^{\operatorname{diam} \Omega}\left(1+\frac{r}{h}\right)^{-3} r^{2} d r \leq c h^{4}|\ln h| \leq c h^{\alpha+5 / 2}, \\
h^{2}\left|\left(f, \chi \widetilde{w}^{2}\right)_{\Omega(h)}\right| \leq c h^{2} \int_{0}^{\operatorname{diam} \Omega}\left(1+\frac{r}{h}\right)^{-2+\delta} r^{2} d r \leq c h^{4-\delta} \leq c h^{\alpha+5 / 2} .
\end{array}
$$


In the estimation (4.30) we have used the formulae (3.32) and (3.59) for $\widetilde{w}^{1}$ and $\widetilde{w}^{2}$ together with the demanded inclusions $\alpha \in(1 / 2,1)$ and $\delta \in(0,1 / 2)$.

Now formulae (4.28), (4.29) and (3.80), (4.25) convert (4.27) into the form

$$
\begin{aligned}
& \mathcal{E}\left(u^{h} ; \Omega(h)\right)-\mathcal{E}(u ; \Omega)=-\frac{1}{2}\left(f, u_{(-)}\right)_{\omega_{h}}+ \\
& +\frac{1}{2} h^{3} f(0)^{\top} \operatorname{mes}_{3} \omega\left(\left(f, G_{(-)}^{0}\right)_{\Omega}+\left(g, G_{(-)}^{0}\right)_{\Gamma_{\sigma}}\right)- \\
& -\frac{1}{2} h^{3}\left(\varepsilon^{0}\right)^{\top} M\left(\left(f, \mathbf{G}_{(-)}^{0}\right)_{\Omega}+\left(g, \mathbf{G}_{(-)}^{0}\right)_{\sigma_{\sigma}}\right)+O\left(h^{\alpha+5 / 2}\right)= \\
& -\frac{1}{2}\left(\left(f, u_{(-)}\right)_{\omega_{h}}-u_{-}(0)^{\top} f(0) m e s_{3} \omega_{h}\right)+\frac{1}{2} h^{3}\left(\varepsilon^{0}\right)^{\top} M \varepsilon_{(-)}^{0}+O\left(h^{\alpha+5 / 2}\right) \\
& =\frac{1}{2} h^{3}\left(\varepsilon^{0}\right)^{\top} M_{(-)} \varepsilon^{0}+O\left(h^{\alpha+5 / 2}\right) .
\end{aligned}
$$

Here, we have taken into account that, first, $M \varepsilon_{(-)}^{0}=M_{(=)} \varepsilon^{0}$ according to the definition of $M_{(=)}$in (3.40) and, second, $u_{-}(0)^{\top} f(0) m_{e} s_{3} \omega_{h}=\left(f, u_{(-)}\right) \omega_{h}+O\left(h^{3+\alpha}\right)$ due to the smoothness properties (3.10) and (3.11) of $f$ and $u$.

Let us formulate the result obtained in (4.32).

Theorem 22 The electrical enthalpy (2.20) admits the asymptotic expansion

$$
\mathcal{E}\left(u^{h} ; \Omega(h)\right)=\mathcal{E}(u ; \Omega)+\frac{1}{2} h^{3}\left(\varepsilon^{0}\right)^{\top} M_{(=)} \varepsilon^{0}+O\left(h^{\alpha+5 / 2}\right),
$$

where $u^{h}$ and $u$ imply solutions of the piezoelectricity problems (3.2)-(3.5) and (2.9)-(2.11), respectively, $\varepsilon^{0}=D\left(\nabla_{x}\right) u(0)$ is the strain column (3.12) and $M_{(=)}=M_{(=)}\left(A^{0}, \omega\right)$ is the modified polarization matrix which is a symmetric matrix of size $9 \times 9$ (see formulae (3.39), (3.40) and Theorem 12).

Note that in contrast to the energy functional (2.16) the electrical enthalpy has the topological derivative

$$
\frac{1}{2}\left(D\left(\nabla_{x}\right) u(0)\right)^{\top} M_{(=)}\left(A^{0}, \omega_{h}\right) D\left(\nabla_{x}\right) u(0)
$$

expressed in terms of local characteristics of the elastic/electric state in the entire body $\Omega$ and of the shape of the small void $\omega_{h}$. Owing to representation (3.41), we emphasize that the polarization matrix (3.39) enjoys the homogeneity property $M\left(A^{0} ; \omega_{h}\right)=h^{3} M\left(A^{0} ; \omega\right)$ which has been used in the passage from (4.33) to (4.34).

Notice, that exponent 3 in the factor $h^{3}$ is conform with formula (3.41) for polarization matrix which contains the volume $\operatorname{mes}_{3} \omega$ of the void.

\subsection{Shape functionals and the adjoint state}

Possible applications of asymptotic analysis performed in the paper include inverse problems, optimum design and shape optimization. We refer the reader to [2, 8, 9, for numerical results of shape and topology optimization by an application of the levelset method. In the levelset method the topological derivative of a specific shape functional is employed to detect the regions of the hold-all domain to include voids in order to improve the value of the functional to be optimized. The numerical method turn out to be very efficient in two spatial dimensions compared to the pure levelset strategy. Another application with, it seems, very high potential for numerical solution are all types of inverse problems to detect imperfections within a geometrical domain on the basis of boundary observations. However, in inverse problems it is required that the data imply the unique identification of the imperfection. This property is unknown, in general, for the strategy which is based on the asymptotic analysis in singularly perturbed domains. In particular, it is an open problem how to identify an imperfection from the observation of a finite number of eigenmodes (eigenvalues), which seems to be a natural and efficient way to solve the problem. The difficulty of such an approach is hidden in the fine properties of topological derivatives which are still to be investigated, for example that some sufficiently large set of observations in the mathematical model based on the asymptotic analysis leads to the uniqueness of the position of imperfection. It means that the derivation of topological derivatives is far from being sufficient for the practical applications of 
the promising tool of shape and topology optimization and identification. We can consider below some specific examples of shape functionals, the other possibilities, including the spectral problem require some additional work to derive the asymptotic formulae.

We return to the analysis. Recalling the Sobolev embedding theorem $H^{1}(\Omega) \subset L^{6}(\Omega)$ in $\mathbb{R}^{3}$, we assume that the density $J$ in the shape functional

$$
\mathcal{J}(u ; \Omega)=\int_{\Omega} J(u(x) ; x) d x
$$

satisfies the following restrictions:

$$
\begin{gathered}
|J(a ; x)| \leq c\left(1+|a|^{t}\right), \\
\left|J(b ; x)-J(a ; x)-J^{\prime}(a ; x)^{\top}(b-a)\right| \leq c|a-b|^{2}\left(1+|a|^{t-2}+|b|^{t-2}\right), \\
|J(b ; x)-J(b ; 0)| \leq c|x|^{\gamma}\left(1+|b|^{t}\right)
\end{gathered}
$$

where $x \in \Omega, a$ and $b$ are arbitrary columns in $\mathbb{R}^{4}$, and the vector function $J^{\prime}$ is subject to the conditions

$$
\begin{gathered}
\left|J^{\prime}(a ; x)\right| \leq c\left(1+|a|^{t-1}\right), \\
\left|J^{\prime}(a ; x)-J^{\prime}(b ; y)\right| \leq c\left(|a-b|^{\gamma}\left(|a|^{t-\gamma}+|b|^{t-\gamma}\right)+|x-y|^{\gamma}\left(|a|^{t}+|b|^{t}\right)\right),
\end{gathered}
$$

while

$$
t \in[2,6), \gamma \in(0,1)
$$

In other words, along with the restrictions on the growth of $J$ and $J^{\prime}$, the integrand $J$ is differentiable with respect to the first variable and Hölder continuous with respect to the second variable. Moreover, $J^{\prime}$ is Hölder continuous in both arguments. Inequality (4.36) ensure that functional (4.35) is defined for $u \in H^{1}(\Omega)^{4} \subset L^{6}(\Omega)^{4} \subset L^{t}(\Omega)^{4}$.

Remark 23 Simple examples

$$
\int_{\Omega(h)} R(x)\left|u^{h}(x)-u(x)\right|^{2} d x \quad \text { and } \quad \int_{\Omega(h)} R(x)\left|u^{h}(x)\right|^{2} d x
$$

are related to the least square method and satisfy the above requirements with $t=2$ and $\gamma=1$ for $R \in C^{1, \alpha}(\Omega)$. In addition, for $g=0$ in the boundary conditions (2.10) and (3.3), the work of external forces

$$
\int_{\Omega(h)} f^{\mathrm{M}}(x)^{\top} u^{h \mathrm{M}}(x) d x+\int_{\Omega(h)} f^{\mathrm{E}}(x)^{\top} u^{h \mathrm{E}}(x) d x
$$

and the electric enthalpy (cf. (4.27)

$$
\mathcal{E}\left(u^{h} ; \Omega(h)\right)=-\frac{1}{2} \int_{\Omega(h)} f^{\mathrm{M}}(x)^{\top} u^{h \mathrm{M}}(x) d x+\frac{1}{2} \int_{\Omega(h)} f^{\mathrm{E}}(x)^{\top} u^{h \mathrm{E}}(x) d x
$$

readily display another examples.

We consider the difference

$$
\mathcal{J}\left(u^{h} ; \Omega(h)\right)-\mathcal{J}(u ; \Omega)=\int_{\Omega(h)}\left(J\left(u^{h}(x) ; x\right)-J(u(x) ; x)\right) d x+\int_{\omega_{h}} J(u(x) ; x) d x .
$$

and, owing to (4.37) and (3.75), obtain the formula

$$
\begin{aligned}
& \left|J\left(u^{h}(x) ; x\right)-J(u(x) ; x)-J^{\prime}(u(x) ; x)^{\top}\left(h^{3} \mathbf{U}(x)+\chi(x) \sum_{j=1}^{2} h^{j} \widetilde{w}^{j}\left(\frac{x}{h}\right)+\widetilde{u}^{h}(x)\right)\right| \leq \\
& \quad \leq c\left(h^{6}|\mathbf{U}(x)|^{2}+\chi(x)^{2} \sum_{j=1}^{2} h^{2 j}\left|\widetilde{w}^{j}\left(\frac{x}{h}\right)\right|^{2}+\left|\widetilde{u}^{h}(x)\right|^{2}\right)\left(1+\left|u^{h}(x)\right|^{t-2}+|u(x)|^{t-2}\right) .
\end{aligned}
$$


Recalling the estimates (4.4), (4.39) and applying the Hölder inequality with the index couples $(p, q)=$ $(5 / 6,6)$ and $(p, q)=(3,2 / 3)$, we obtain

$$
\begin{aligned}
& \int_{\Omega(h)} J^{\prime}(u(x) ; x)^{\top} \widetilde{u}^{h}(x) d x \leq c \int_{\Omega(h)}\left(1+|u(x)|^{5}\right)\left|\widetilde{u}^{h}(x)\right| d x \leq \\
& \leq c\left(1+\left\|u ; L^{6}(\Omega)\right\|^{5}\right)\left\|\widetilde{u}^{h} ; L^{6}(\Omega(h))\right\| \leq c\left\|\widetilde{u}^{h} ; H^{1}(\Omega)\right\| \leq c h^{\alpha+5 / 2}, \\
& \int_{\Omega(h)}\left|\widetilde{u}^{h}\right|^{2}\left(1+\left|u^{h}\right|^{t-2}+|u|^{t-2}\right) d x \leq c \int_{\Omega(h)}\left|\widetilde{u}^{h}\right|^{2}\left(1+\left|u^{h}\right|^{4}+|u|^{4}\right) d x \leq \\
& \leq c\left\|\widetilde{u}^{h} ; L^{6}(\Omega(h))\right\|^{2}\left(1+\left\|u^{h} ; L^{6}(\Omega(h))\right\|^{4}+\left\|u ; L^{6}(\Omega)\right\|^{4}\right) \leq c h^{2 \alpha+5} .
\end{aligned}
$$

Similarly,

$$
h^{6} \int_{\Omega \backslash \mathbb{B}_{R}^{\prime}}|\mathbf{U}(x)|^{2}\left(1+\left|u^{h}(x)\right|^{t-2}+|u(x)|^{t-2}\right) d x \leq c h^{6} .
$$

However, because of the singularity $|\mathbf{U}(x)|=O\left(|x|^{-2}\right)$, we use in the ball $\mathbb{B}_{R^{\prime}}$ the Hölder inequality with the couple

$$
(p, q)=\left(\frac{6}{8-t}, \frac{6}{t-2}\right)
$$

to derive that

$$
\begin{aligned}
h^{6} \int_{\mathbb{B}_{R^{\prime}} \backslash \omega_{h}}|\mathbf{U}|^{2}\left(1+\left|u^{h}\right|^{t-2}+|u|^{t-2}\right) d x \leq c h^{6}\left(\int_{c h}^{R^{\prime}} r^{-\frac{24}{8-t}} r^{2} d r\right)^{\frac{8-t}{6}} \times \\
\quad \times\left(1+\left\|u^{h} ; H^{1}(\Omega(h))\right\|^{t-2}+\left\|u ; H^{1}(\Omega)\right\|^{t-2}\right) \leq c h^{6-t / 2} .
\end{aligned}
$$

We deal with the boundary layers in the same way as in (4.15) and (4.16). Outside the ball $\mathbb{B}_{R h}$ we apply the inequalities (3.32) and (3.59) even much rougher ones, to conclude by the Hölder inequality with the index couple (4.44) that

$$
\begin{aligned}
h^{2 j} \int_{\Omega \backslash \mathbb{B}_{R h}} & \left|\chi(x) \widetilde{w}^{j}\left(\frac{x}{h}\right)\right|^{2}\left(1+\left|\widetilde{u}^{h}(x)\right|^{2}+|u(x)|^{2}\right) d x \leq \\
& \leq \operatorname{ch}^{6}\left(\int_{c h}^{R^{\prime}} r^{-\frac{12(3-j)}{8-t}} r^{2} d r\right)^{\frac{8-t}{6}} \leq \operatorname{ch}^{6-t / 2}, j=1,2 .
\end{aligned}
$$

Inside the ball $\mathbb{B}_{R h}$ the Hölder inequality gives

$$
\begin{aligned}
h^{2 j} \int_{\mathbb{B}_{R h} \backslash \omega_{h}} & \left|\widetilde{w}^{j}\left(\frac{x}{h}\right)\right|^{2}\left(1+\left|\widetilde{u}^{h}(x)\right|^{2}+|u(x)|^{2}\right) d x \\
& \leq c h^{2 j}\left(\int_{\substack{\mathbb{B}_{R h} \backslash \omega_{h}\\
}}\left|\widetilde{w}^{j}\left(\frac{x}{h}\right)\right|^{\frac{12}{8-t}} d x\right)^{\frac{8-t}{6}}=c h^{2 j+3 \frac{8-t}{6}}\left(\left.\int_{\mathbb{B}_{R h} \backslash \omega} \widetilde{w}^{j}\left(\frac{x}{h}\right)\right|^{\frac{12}{8-t}} d \xi\right)^{\frac{8-t}{6}} \\
& \leq c h^{2 j+4-t / 2} \leq c h^{6-t / 2}, j=1,2 .
\end{aligned}
$$

Note that $\frac{12}{8-t}<6$ due to (4.41) and, therefore,

$$
\left\|\widetilde{w}^{j} ; L^{\frac{12}{8-t}}\left(\mathbb{B}_{R} \backslash \omega\right)\right\| \leq c\left\|\widetilde{w}^{j} ; H^{1}\left(\mathbb{B}_{R} \backslash \omega\right)\right\| \leq c\left\|\widetilde{w}^{j} ; V_{0}^{1}(\Xi)\right\| .
$$


Although, the faster rates of decay of the remainders $\widetilde{w}^{1}$ and $\widetilde{w}^{2}$ (cf. (3.66) ) are not used in the estimation (4.45), the rate of decay becomes an important ingredient of the inequalities

$$
h^{j}\left|\int_{\Omega(h)} J^{\prime}(u(x) ; x)^{\top} \chi(x) \widetilde{w}^{j}\left(\frac{x}{h}\right) d x\right| \leq c h^{7 / 2}, j=1,2,
$$

its derivation is much simpler, though. A simplification originates from the relation $\left|J^{\prime}(u(x) ; x)\right| \leq$ const for $x \in \operatorname{supp} \chi \subset \mathbb{B}_{R^{\prime}}$ so that one may repeat the calculation (4.30).

Finally, we write

$$
h^{3}\left|\int_{\omega_{h}} J^{\prime}(u(x) ; x)^{\top} \mathbf{U}(x) d x\right| \leq c h^{3} \int_{0}^{R h} r^{-2} r^{2} d r \leq c h^{4}
$$

and, in view of (3.11) and (4.40),

$$
\left|\int_{\omega_{h}} J(u(x) ; x) d x-h^{3} J(u(0) ; 0) \operatorname{mes}_{3} \omega\right| \leq \operatorname{ch}^{3+\min \{\alpha, \gamma\}}
$$

Everything is prepared to derive a formula of type (2.1) for the shape functional (4.35).

Theorem 24 Let the assumption formulated above hold true. Then the asymptotic formula

$$
\begin{aligned}
\mathcal{J}\left(u^{h} ; \Omega(h)\right) & =\mathcal{J}(u ; \Omega)+h^{3}\left(\left(J(u(0) ; 0)-P(0)^{\top} f(0)\right) \operatorname{mes}_{3} \omega\right. \\
& \left.-\left(D\left(\nabla_{x}\right) P(0)\right)^{\top} M \varepsilon^{0}\right)+O\left(h^{3+\min \{\gamma, \alpha-1 / 2,3-t / 2\}}\right)
\end{aligned}
$$

is valid where $P \in \stackrel{\circ}{H}^{1}\left(\Omega ; \Gamma_{u}\right)^{4} \cap C^{2, \min }\{\alpha, \gamma\}\left(\mathbb{B}_{R^{\prime}}\right)^{4}$ is a solution of the formally adjoint piezoelectricity problem

$$
\begin{aligned}
& D\left(-\nabla_{x}\right)^{\top} A(x)^{\top} D\left(\nabla_{x}\right) P(x)=J^{\prime}(u(x) ; x), x \in \Omega, \\
& D(n(x))^{\top} A(x)^{\top} D\left(\nabla_{x}\right) P(x)=0, x \in \Gamma_{\sigma}, P(x)=0, x \in \Gamma_{\sigma} .
\end{aligned}
$$

Proof. The calculations performed above provide the relation

$$
h^{-3}\left(\mathcal{J}\left(u^{h} ; \Omega(h)\right)-\mathcal{J}(u ; \Omega)\right)=J(u(0) ; 0) \text { mes }_{3} \omega+\left(J^{\prime}(u), \mathbf{U}\right)_{\Omega}+O\left(h^{\min \{\gamma, \alpha-1 / 2,3-t / 2\}}\right) .
$$

We recall the representation (3.80) where $G^{0}$ is the Green matrix, i.e., a solution to the problem (3.77). The Green matrix and its derivatives help to calculate the solution $P$ of the formally adjoint problem (4.47) and the derivatives $\mathbf{G}^{0}$ (see (3.78)) deliver the column $D\left(\nabla_{x}\right) P(x)$ at the point $x=0$. In other words, we write

$$
\begin{aligned}
\left(J^{\prime}(u), \mathbf{U}\right)_{\Omega} & =\left(D\left(-\nabla_{x}\right)^{\top} A^{\top} D\left(\nabla_{x}\right) P, \mathbf{G}^{0}\right)_{\Omega} M \varepsilon^{0} \\
& -\operatorname{mes}_{3} \omega\left(D\left(-\nabla_{x}\right)^{\top} A^{\top} D\left(\nabla_{x}\right) P, G^{0}\right)_{\Omega} f(0) \\
& =\left(P, D\left(\nabla_{x}\right)^{\top} \delta M \varepsilon^{0}\right)_{\Omega}-m e s_{3} \omega(P, \delta f(0))_{\Omega} \\
& =-\left(D\left(\nabla_{x}\right) P(0)\right)^{\top} M \varepsilon^{0}-P(0)^{\top} f(0) m_{3} \omega .
\end{aligned}
$$

We again used the Dirac mass $\delta$ in the framework of the theory of distributions to compute the expression (4.48).

Finally, in order to justify our calculations we make the following comments. By assumptions (4.36), (4.41) and (4.40), (3.9), the functional

$$
\stackrel{\circ}{H}^{1}\left(\Omega ; \Gamma_{u}\right)^{4} \ni v \rightarrow\left(J^{\prime}(u), v\right)_{\Omega}
$$

is continuous and $J^{\prime}(u) \in C^{0, \min \{\alpha, \gamma\}}\left(\mathbb{B}_{R^{\prime}}\right)$ with any $R^{\prime}<R$. Thus, the same arguments as in Sections 2.4 and 3.1 guarantee the existence of a solution $P$ to the problem (4.47) which is twice differentiable in the vicinity of the point $x=0$. These observations make all calculations justified.

The topological derivative of the functional $\mathcal{J}$, i.e.,

$$
\mathcal{T}(u, \omega)=\left(J(u(0) ; 0)-P(0)^{\top} f(0)\right) \operatorname{mes}_{3} \omega-\left(D\left(\nabla_{x}\right) P(0)\right)^{\top} M \varepsilon^{0},
$$

is non-local since it involves the adjoint state $P$ in (4.47) which depends on the solution $u$ of the piezoelectricity problem in the entire domain $\Omega$. 


\subsection{Example}

It turns out, that the so-called weak interaction is quite common feature of piezoelectric materials. Therefore, we are going to present an example for such materials. Assume that there is a weak interaction between the mechanical and electric fields. This means that in the decomposition

$$
\begin{gathered}
A=A_{(0)}+A_{(1)}, \\
A_{(0)}=\left(\begin{array}{cc}
A^{\mathrm{MM}} & \mathbb{O}_{6 \times 3} \\
\mathbb{O}_{3 \times 6} & A^{\mathrm{EE}}
\end{array}\right), \quad A_{(1)}=\left(\begin{array}{cc}
\mathbb{O}_{6 \times 6} & -A^{\mathrm{ME}} \\
A^{\mathrm{EM}} & \mathbb{O}_{3 \times 3}
\end{array}\right)
\end{gathered}
$$

the entries of matrix $A_{(1)}$ are much smaller compared to non trivial entries of the matrix $A_{(0)}$. It implies that in the first order approximation the piezoelectricity problem is decoupled into two problems, the pure elasticity problem with the stiffness matrix $A^{\mathrm{MM}}$, and the pure electricity problem with the permeability matrix $A^{\mathrm{EE}}$.

We are going to evaluate the main correction terms in the asymptotic expansions of characteristics for the piezoelectric bodies $\Omega, \Xi$ and $\Omega(h)$ (see Sections 2.3, 3.3 and 3.1). We point out that to evaluate the main asymptotic terms in all formulae given below, it is sufficient to solve only the pure elasticity and the pure electricity problems.

Remark 25 Since we always deal with the first order asymptotic corrections, the introduction of any small amplitude parameter neither makes formulae more transparent, nor contribute to the exactness of presentation. We emphasize that, in contrast to the preceding sections, the perturbations here are of regular type, which means that the justification of obtained formulae relies upon the standard argument of convergence of Neumann series. In order to simplify the notation, in the sequel the second order terms are always denoted by dots, starting from (4.50).

We proceed with the solution

$$
u(x)=u_{(0)}+u_{(1)}(x)+\ldots
$$

of the problem (2.9)-(2.11). In view of (4.49), the displacement vector $u_{(0)}^{\mathrm{M}}$ and the electric vector $u_{(0)}^{\mathrm{E}}$ verify the problems

$$
\begin{gathered}
D^{\mathrm{M}}\left(-\nabla_{x}\right)^{\top} A^{\mathrm{MM}}(x) D^{\mathrm{M}}\left(\nabla_{x}\right) u_{(0)}^{\mathrm{M}}(x)=f^{\mathrm{M}}(x), x \in \Omega, \\
D^{\mathrm{M}}(n(x))^{\top} A^{\mathrm{MM}}(x) D^{\mathrm{M}}\left(\nabla_{x}\right) u_{(0)}^{\mathrm{M}}(x)=g^{\mathrm{M}}(x), x \in \Gamma_{\sigma}, \quad u_{(0)}^{\mathrm{M}}(x)=0, x \in \Gamma_{u}, \\
-\nabla_{x}^{\top} A^{\mathrm{EE}} \nabla_{x} u_{(0)}^{\mathrm{E}}(x)=f^{\mathrm{E}}(x), x \in \Omega, \\
n^{\top} A^{\mathrm{EE}} u_{(0)}^{\mathrm{E}}(x)=g^{\mathrm{E}}(x), x \in \Gamma_{\sigma}, \quad u_{(0)}^{\mathrm{E}}(x)=0, x \in \Gamma_{u},
\end{gathered}
$$

and can be determined separately. Inserting (4.50) and (4.49) into (2.9) - (2.11), we arrive at the problem

$$
\begin{gathered}
D\left(-\nabla_{x}\right)^{\top} A_{(0)}(x) D\left(\nabla_{x}\right) u_{(1)}(x)=D\left(-\nabla_{x}\right)^{\top} A_{(1)}(x) D\left(\nabla_{x}\right) u_{(0)}(x), x \in \Omega, \\
D(n(x))^{\top} A_{(0)}(x) D\left(\nabla_{x}\right) u_{(1)}(x)=D(n(x))^{\top} A_{(1)}(x) D\left(\nabla_{x}\right) u_{(0)}(x), x \in \Gamma_{\sigma}, \\
u_{(1)}(x)=0, x \in \Gamma_{u} .
\end{gathered}
$$

This problem is decoupled as well, however, its solution manifests the interaction between electric and mechanical fields, since the displacement vector $u_{(1)}^{\mathrm{M}}$ depends only on the main part $u_{(0)}^{\mathrm{E}}$ of the electric potential and, in the same manner, $u_{(1)}^{\mathrm{E}}$ depends on $u_{(0)}^{\mathrm{M}}$.

In order to complete the asymptotic formulae, in the same way as in the previous sections, we also need the expansion for the polarization matrix 


$$
\begin{gathered}
M=M_{(0)}+M_{(1)}+\ldots \\
M_{(0)}=\left(\begin{array}{cc}
M_{(0)}^{\mathrm{M}} & \mathbb{O}_{6 \times 3} \\
\mathbb{O}_{3 \times 6} & M_{(0)}^{\mathrm{E}}
\end{array}\right), \quad M_{(1)}=\left(\begin{array}{cc}
\mathbb{O}_{6 \times 6} & M_{(1)}^{\mathrm{ME}} \\
M_{(1)}^{\mathrm{EM}} & \mathbb{O}_{3 \times 3}
\end{array}\right) .
\end{gathered}
$$

We emphasize that the matrices $M_{(0)}$ and $M_{(1)}$ inherit the block diagonal structure of $A_{(0)}$ and the block-anti-diagonal of $A_{(1)}$, respectively. The same structures are kept by all matrix objects, in particular, the fundamental matrix takes the form

$$
\begin{gathered}
\Phi=\Phi_{(0)}+\Phi_{(1)}+\ldots, \\
\Phi_{(0)}=\left(\begin{array}{cc}
\Phi_{(0)}^{\mathrm{M}} & \mathbb{O}_{3 \times 1} \\
\mathbb{O}_{1 \times 3} & \Phi_{(0)}^{\mathrm{E}}
\end{array}\right), \quad \Phi_{(1)}=\left(\begin{array}{cc}
\mathbb{O}_{3 \times 3} & \Phi_{(1)}^{\mathrm{ME}} \\
\Phi_{(1)}^{\mathrm{EM}} & 0
\end{array}\right) .
\end{gathered}
$$

Here, $\Phi_{(0)}^{\mathrm{M}}$ is the fundamental matrix for the elasticity matrix operator $D^{\mathrm{M}}\left(-\nabla_{\xi}\right)^{\top} A^{0 \mathrm{M}} D^{\mathrm{M}}\left(\nabla_{\xi}\right)$ and $\Phi_{(0)}^{\mathrm{E}}$ is the fundamental matrix for the scalar operator $-\nabla_{\xi}^{\top} A^{0 \mathrm{E}} \nabla_{\xi}$. Furthermore, $M_{(0)}^{\mathrm{E}}$ and $M_{(0)}^{\mathrm{M}}$ are the virtual mass matrix and the elasticity polarization matrix for the cavity $\omega \subset \mathbb{R}^{3}$, which are negative definite (see 48 and 38, 44).

It is convenient to proceed with the matrix solution (3.37) which, according to (4.49) and (3.38), enjoys the expansion

$$
\begin{gathered}
W=W_{(0)}+W_{(1)}+\ldots \\
W_{(0)}=\left(\begin{array}{cc}
W_{(0)}^{\mathrm{M}} & \mathbb{O}_{3 \times 3} \\
\mathbb{O}_{1 \times 6} & W_{(0)}^{\mathrm{E}}
\end{array}\right), \quad W_{(1)}=\left(\begin{array}{cc}
\mathbb{O}_{3 \times 6} & W_{(1)}^{\mathrm{ME}} \\
W_{(1)}^{\mathrm{EM}} & \mathbb{O}_{1 \times 3}
\end{array}\right)
\end{gathered}
$$

with

$$
\begin{gathered}
W(\xi)=\left(M D\left(\nabla_{\xi}\right) \Phi(\xi)^{\top}\right)^{\top}+O\left(|\xi|^{-2}\right)= \\
\left(M_{(0)} D\left(\nabla_{\xi}\right) \Phi_{(0)}(\xi)^{\top}\right)^{\top}+\left(M_{(0)} D\left(\nabla_{\xi}\right) \Phi_{(1)}(\xi)^{\top}+M_{(1)} D\left(\nabla_{\xi} \Phi_{(0)}(\xi)^{\top}\right)^{\top}+\cdots+O\left(|\xi|^{-2}\right) .\right.
\end{gathered}
$$

The correction term $\Phi_{(1)}$ in (4.55) is a power-law solution of form (3.53) for the system of differential equations

$$
D\left(-\nabla_{\xi}\right)^{\top} A_{(0)}^{0} D\left(\nabla_{\xi}\right) \Phi_{(1)}(\xi)=D\left(\nabla_{\xi}\right)^{\top} A_{(1)}^{0} D\left(\nabla_{\xi}\right) \Phi_{(0)}(\xi), \quad \xi \in \mathbb{R}^{3} \backslash\{0\},
$$

(cf. (3.54)). By a general result in 20] (see also [39, Lemmas 3.3.1 and 3.5.11]), the solution $\Phi_{(1)}$ can depend linearly on $\ln |\xi|$, however, the same argument as in the proof of Lemma 14 ensures that $\Phi_{(1)}$ is positive homogeneous of degree -1 according to 3.30). The solution $\Phi_{(1)}$, which is defined up to the linear combination $\Phi_{(0)} C$ of the fundamental matrix columns with the constant column $C \in \mathbb{R}^{4}$, can be fixed such that

$$
\int_{\mathbb{S}_{\varkappa}} D\left(\nabla_{\xi}\right)^{\top} A^{0} D\left(\nabla_{\xi}\right) \Phi_{(1)}(\xi) d s_{\xi}=0 \in \mathbb{R}^{4} .
$$

The exterior problem for the correction term in (4.56) takes the form

$$
\begin{gathered}
D\left(-\nabla_{\xi}\right)^{\top} A_{(0)}^{0} D\left(\nabla_{\xi}\right) W_{(1)}(\xi)=D\left(\nabla_{\xi}\right)^{\top} A_{(1)}^{0} D\left(\nabla_{\xi}\right) W_{(0)}(\xi), \xi \in \Xi, \\
D\left(n^{\omega}(\xi)\right)^{\top} A_{(0)}^{0} D\left(\nabla_{\xi}\right) W_{(1)}(\xi)=-D\left(n^{\omega}(\xi)\right)^{\top} A_{(1)}^{0} D\left(\nabla_{\xi}\right) W_{(0)}(\xi), \xi \in \partial \omega .
\end{gathered}
$$


Since, owing to (4.57), we have

$$
W_{(0)}(\xi)=\left(M_{(0)} D\left(\nabla_{\xi}\right) \Phi_{(0)}(\xi)^{\top}\right)^{\top}+O\left(|\xi|^{-3}\right),
$$

the right-hand side $F_{(1)}(\xi)$ in (4.60) admits the decomposition

$$
\begin{gathered}
F_{(1)}(\xi)=D\left(\nabla_{\xi}\right)^{\top} A_{(1)}^{0} D\left(\nabla_{\xi}\right)\left(D\left(\nabla_{\xi}\right) \Phi_{(0)}(\xi)^{\top}\right)^{\top} M_{(0)}^{\top}+\widetilde{F}_{(1)}(\xi)= \\
=\sum_{q=1}^{3} \frac{\partial}{\partial \xi_{q}} D\left(\nabla_{\xi}\right)^{\top} A_{(1)}^{0} D\left(\nabla_{\xi}\right) \Phi_{(0)}(\xi)^{\top} D\left(e_{q}\right)^{\top} M_{(0)}^{\top}+\widetilde{F}_{(1)}(\xi)= \\
=\sum_{q=1}^{3} D\left(-\nabla_{\xi}\right)^{\top} A_{(0)}^{0} D\left(\nabla_{\xi}\right) \frac{\partial \Phi_{(1)}}{\partial \xi_{q}} D\left(e_{q}\right)^{\top} M_{(0)}^{\top}+\widetilde{F}_{(1)}(\xi)
\end{gathered}
$$

with the remainder $\widetilde{F}_{(1)}(\xi)=O\left(|\xi|^{-5}\right)$. In (4.62), the equation (4.58) has been applied. Comparing (4.62) with (4.57), we set

$$
W_{(1)}(\xi)=\widetilde{W}_{(1)}(\xi)+\left(M_{(0)} D\left(\nabla_{\xi}\right) \Phi_{(0)}(\xi)^{\top}\right)^{\top} .
$$

Recall that $\omega$ contains the origin $\xi=0$, therefore, the last term in (4.63) is smooth in $\bar{\Xi}$. As a result, a new exterior problem is obtained, with the right-hand side $\widetilde{F}_{(1)}$ which decays sufficiently fast at infinity,

$$
\begin{gathered}
D\left(-\nabla_{\xi}\right)^{\top} A_{(0)}^{0} D\left(\nabla_{\xi}\right) \widehat{W}_{(1)}(\xi)=\widetilde{F}_{(1)}(\xi), \xi \in \Xi, \\
D\left(n^{\omega}(\xi)\right)^{\top} A_{(0)}^{0} D\left(\nabla_{\xi}\right) \widehat{W}_{(1)}(\xi)=\widetilde{G}_{(1)}(\xi), \xi \in \partial \omega,
\end{gathered}
$$

where

$$
\begin{gathered}
\widetilde{G}_{(1)}(\xi)=D\left(n^{\omega}(\xi)\right)^{\top} A_{(1)}^{0} D\left(\nabla_{\xi}\right) \widehat{W}_{(0)}(\xi)- \\
-D\left(n^{\omega}(\xi)\right)^{\top} A_{(0)}^{0} D\left(\nabla_{\xi}\right)\left(M_{(0)} D\left(\nabla_{\xi}\right) \Phi_{(1)}(\xi)^{\top}\right)^{\top} .
\end{gathered}
$$

Now, the decay of $\widetilde{G}_{(1)}(\xi)$ can be used, indeed, by Proposition 8 (see [20] and [39, Theorem 3.5.6]) and the calculations (3.34), (3.33), the solution $\widehat{W}_{(1)} \in V_{0}^{1}(\Xi)^{4}$ admits the asymptotic form

$$
\widehat{W}_{(1)}(\xi)=\left(M_{(1)} D\left(\nabla_{\xi}\right) \Phi_{(0)}(\xi)^{\top}\right)^{\top}+\widetilde{W}_{(1)}(\xi),
$$

where the remainder $\widetilde{W}_{(1)}$ is subject to the estimates (3.32) with the majorants $c_{k} \rho^{-3-k+\delta}(\delta>0$ is arbitrary) and the notation used for the derivatives of the fundamental matrix $\Phi_{(0)}$ is matched with formulae (4.57) and (4.63).

In order to evaluate the correction term $M_{(1)}$ in the expansion of the polarization matrix the method 29 is employed, here we recall that the columns of the matrix

$$
\mathcal{W}_{(0)(-)}(\xi)=D_{(-)}(\xi)^{\top}+W_{(0)(-)}(\xi)
$$

(cf. (3.41)) are formal solutions to the homogeneous problem (4.64). By the Green formula in $\Xi \cap \mathbb{B}_{R}$, we obtain

$$
\begin{gathered}
\int_{\Xi \cap \mathbb{B}_{R}} \mathcal{W}_{(0)(-)}(\xi)^{\top} \widetilde{F}_{(1)}(\xi) d \xi+\int_{\partial \omega} \mathcal{W}_{(0)(-)}(\xi)^{\top} \widetilde{G}_{(1)}(\xi) d s_{\xi}= \\
\int_{\partial \mathbb{B}_{R}}\left(\widehat{W}_{(1)}(\xi)^{\top} D\left(|\xi|^{-1} \xi\right)^{\top} A_{(0)(-)}^{0} D(\xi) \mathcal{W}_{(0)(-)}-\mathcal{W}_{(0)(-)}^{\top} D\left(|\xi|^{-1} \xi\right)^{\top} A_{(0)(-)}^{0} D(\xi) \widehat{W}_{(1)}(\xi)\right) d s_{\xi} \\
+O\left(R^{-1}\right)=-M_{(1)(=)}+O\left(R^{-1}\right)
\end{gathered}
$$


We have here repeated the computation (3.45) based on the representations (4.66) and (4.67). The integrand on the left-hand side of (4.68) is of order $|\xi|^{-4}$ and, hence, the integral over $\Xi$ converges and the formula

$$
M_{(1)(=)}=\left(\begin{array}{cc}
\mathbb{O}_{6 \times 6} & M_{(1)}^{\mathrm{ME}} \\
M_{(1)}^{\mathrm{EM}} & \mathbb{O}_{3 \times 3}
\end{array}\right)=-\int_{\Xi} \mathcal{W}_{(0)(-)}(\xi)^{\top} \widetilde{F}_{(1)}(\xi) d \xi+\int_{\partial \omega} \mathcal{W}_{(0)(-)}(\xi)^{\top} \widetilde{G}_{(1)}(\xi) d s_{\xi}
$$

together with (4.62)-(4.64) expresses the matrix $M_{(1)}$ (cf. the definition (3.40)) in terms of the matrix $A^{\mathrm{ME}}=\left(A^{\mathrm{EM}}\right)^{-1}$ and the special solutions $W^{1}, \ldots, W^{6}$ and $W^{7}, W^{8}, W^{9}$ of the pure elasticity and the pure electricity exterior problems in $\Xi$. Theorem 12 shows that $\left(M_{(1)}^{\mathrm{ME}}\right)^{\top}=-M_{(1)}^{\mathrm{EM}}$.

The formulae derived above can be used, e.g., to obtain the topological derivative of the electric enthalpy (4.33):

$$
\begin{gathered}
\mathcal{T}_{\mathcal{E}}(u ; \omega)= \\
=\frac{1}{2} h^{3}\left(\left(D^{\mathrm{M}}\left(\nabla_{x}\right) u_{(0)}^{\mathrm{M}}(0)\right)^{\top} M_{(0)}^{\mathrm{M}} D^{\mathrm{M}}\left(\nabla_{x}\right) u_{(0)}^{\mathrm{M}}(0)-\nabla_{x} u_{(0)}^{\mathrm{E}}(0)^{\top} M_{(0)}^{\mathrm{E}} \nabla_{x} u_{(0)}^{\mathrm{E}}(0)\right)+ \\
+h^{3}\left(\left(D^{\mathrm{M}}\left(\nabla_{x}\right) u_{(0)}^{\mathrm{M}}(0)\right)^{\top} M_{(0)}^{\mathrm{M}} D^{\mathrm{M}}\left(\nabla_{x}\right) u_{(1)}^{\mathrm{M}}(0)-\nabla_{x} u_{(0)}^{\mathrm{E}}(0)^{\top} M_{(0)}^{\mathrm{E}} \nabla_{x} u_{(1)}^{\mathrm{E}}(0)\right)+ \\
\left.+h^{3} \nabla_{x} u_{(0)}^{\mathrm{E}}(0)\right)^{\top} M^{\mathrm{EM}} D^{\mathrm{M}}\left(\nabla_{x}\right) u_{(1)}^{\mathrm{M}}(0)+\ldots,
\end{gathered}
$$

where $M_{(0)}^{\mathrm{M}}$ and $M_{(0)}^{\mathrm{E}}$ are the elasticity polarization matrix and the virtual mass matrix for the cavity while $M_{(1)}^{\mathrm{EM}}=-\left(M_{(1)}^{\mathrm{ME}}\right)^{\top}$ is expressed in (4.69).

Even the main term (with the factor $\frac{1}{2} h^{3}$ ) of the topological derivative (4.70) has no sign, that is, in contrast to the forms of topological derivatives of the energy functionals for the pure elasticity and the pure electricity problems. The correction term (with factor $h^{3}$ ) in (4.70) depends on two specific ingredients, namely, the correction term $M^{\mathrm{EM}}$ in polarization matrix (see (4.54) and (4.69)), and the correction terms $u_{(1)}^{\mathrm{M}}, u_{(1)}^{\mathrm{E}}$ for the combined mechanical and electric fields.

Remark 26 All the attributes in the above formulae can be given explicitly for some canonical shapes, including balls, ellipsoids and elliptic cracks in three spatial dimensions, and some other shapes in two spatial dimensions (see [48] and [53, 32, 25, 3] and others).

Remark 27 The case of $g^{\mathrm{E}}=0, f^{\mathrm{E}}=0$ has a very clear physical meaning (i.e. one gets an electric sparkle when pressing the lighter button). Then, in notation of Section 4.2.

$$
\mathfrak{u}^{\mathrm{M}}=u, \quad \mathfrak{u}^{\mathrm{E}}=0, \quad \mathfrak{e}^{\mathrm{M}}=\varepsilon^{0}, \quad \mathfrak{e}^{\mathrm{E}}=0,
$$

thus, by relation (3.40), we can conclude that the topological derivatives in (3.40) and (3.46) of the energy and electric enthalpy functionals coincides one with another. In general, this identity is false, and can be misleading for the choice of governing Gibbs' functional for piezoelectric body (cf. Remark [21). The relations between the topological derivatives for elasticity and piezoelectricity are easy to established, since the topological derivative for piezoelectricity can be viewed as the difference of that for elasticity and of the other for electricity.

Acknowledgements. This paper was prepared during the visit of S.A. Nazarov to the Institute Elie Cartan of the University Henri Poincaré Nancy 1 and to Department of Civil Engineering of Second University of Naples. The research of S.A.N. is partially supported by the grant RFFI-09-01-00759 and by project "Asymptotic analysis of composite materials and thin and non-homogeneous structures" (Regione Campania, law n.5/2006); J.S. was partially supported by the Projet CPER Lorraine MISN: Analyse, optimisation et contrôle 3 in France, and the grant N51402132/3135 Ministerstwo Nauki i Szkolnictwa Wyzszego: Optymalizacja z wykorzystaniem pochodnej topologicznej dla przeplywow w osrodkach scisliwych in Poland. 


\section{References}

[1] Agmon S., Douglis A., Nirenberg L. Estimates near the boundary for solutions of elliptic partial differential equations satisfying general boundary conditions. II. Comm. Pure Appl. Math. 17, 1964, $35-92$.

[2] Allaire G., De Gournay F., Jouve F., Toader A.M., Structural optimization using topological and shape sensitivity via a level set method, Control and Cybernetics (2005).

[3] Ammari H., Kang H. Polarization and moment tensors. With applications to inverse problems and effective medium theory. Applied Mathematical Sciences, 162, Springer, New York, 2007.

[4] Cherkaev A., Gibiansky L. Variational principles for complex conductivity, viscoelasticity and similar problems in media with complex moduli. J. of Math. Physics 35 (1), 1994, 1-19.

[5] Choi K.K., Nam-Ho Kim Structural sensitivity analysis and optimization. volumes 1 and 2, Springer Mechanical Engineering Series, Springer New York, 2005

[6] Delfour M.C., Zolesio J.P., Shapes and Geometries, Advances in Design and Control. Society for Industrial and Applied Mathematics (SIAM), Philadelphia, PA, 2001.

[7] Duvaut G., Lions J.L. Inequalities in Mechanics and Physics. Grundlehren der mathematischen Wissenschaften 219, 1976, Springer-Verlag, Berlin.

[8] Fulmanski P., Lauraine A., Scheid J.F., SokoŁowski J. A level set method in shape and topology optimization for variational inequalities, Int. J. Appl. Math. Comput. Sci., 17, (3) 2007, 413-430.

[9] Fulmanski P., Lauraine A., Scheid J.F., SokoŁowski J. Level set method with topological derivatives in shape optimization International Journal of Computer Mathematics, Vol. 85, No. 10, 2008, 1491-1514.

[10] Garreau S., Guillaume Ph., Masmoudi M. The Topological Asymptotic for PDE Systems: The Elasticity Case. SIAM Journal on Control and Optimization, 39, 2001, 1756-1778.

[11] Gel'fand I.M., Shilov G.E. Generalized functions. Vol. 2: Spaces of fundamental and generalized functions. New York and London, Academic Press. X, 1968.

[12] Grinchenko V.T., Ulitko A.F., Szulga N.A. Mechanics of interacting fields in constructions components Kiev, Naukovaja Dumka, 1989, 280 p. (Russian)

[13] Hörmander, L. Linear partial differential operators. (English) Die Grundlehren der mathematischen Wissenschaften. 116, 1963, Berlin- Gttingen-Heidelberg: Springer-Verlag.

[14] Hlaváček I., Novotny A.A., SokoŁowski J., Żochowski A. On topological derivatives for elastic solids with uncertain input data JOTA, 2009, electronic.

[15] Ikeda T. Fundamentals of Piezoelectricity, Oxford University Press, London, 1996.

[16] IL'IN A.M. Matching of asymptotic expansions of solutions of boundary value problems Moscow: Nauka, [in Russian], 1989. [English translation: Matching of asymptotic expansions of solutions of boundary value problems Translations of Mathematical Monographs, 102, American Mathematical Society, Providence, RI, 1992].

[17] Jikov V. V., Kozlov S. M., Oleinik O. A. Homogenization of differential operators and integral functionals. Springer-Verlag, Berlin, 1994, 570 pp. 
[18] Kaltenbacher B., Lahmer T., Mohr M., Kaltenbacher M. PDE based determination of piezoelectric material tensors Euro J. of Appl. Mathematics 17, 2006, 383-416.

[19] Kapitonov B., Miara B., Perla Menzala G. Boundary observation and exact control of a quasi-electrostatic piezoelectric system in multilayered media SIAM Journal on Control and Optimization, 46, (3) 2007, 1080-1097.

[20] Kondratiev V.A. Boundary problems for elliptic equations in domains with conical or angular points Trudy Moskov. Mat.Obshch., 16, 1967, 209-292. (Engl. transl. in Trans. Moscow Math. Soc., $16,1967,227-313)$.

[21] Kondratiev V.A., Oleinik O.A. Boundary-value problems for the system of elasticity theory in unbounded domains. Korn's inequalities. Russian Mathematical Surveys, 43, (5) 1988, 65-119.

[22] Kulikov A.A., Nazarov S.A. Cracks in piezoelectric and electro-conductive bodies. Siberian J. of Industrial Mathematics, 8, (1) 2005, 70-87. (Engl. transl.: J. Math. Sci., 1, (2) 2007, 201-216).

[23] Ladyzhenskaya O.A. Boundary value problems of mathematical physics Moscow: Nauka, 1973. English translation: The boundary value problems of mathematical physics. Applied Mathematical Sciences, 49, Springer-Verlag, New York, 1985.

[24] Lekhnitskit S. G. Theory of Elasticity of an Anisotropic Elastic Body. Holden-Day, Inc., San Francisco, California, 1963.

[25] Lewinski T., SokoŁowski L. Energy change due to the appearance of cavities in elastic solids. Int. J. Solids Struct. 40, 2003, 1765-1803.

[26] Lipton R. Inequalities for electric and elastic polarization tensors with applications to random composites. J. Mech. Phys. Solids, 5(41), 1993, 809-833.

[27] MaZ'Ya V.G., NaZARov S.A. The asymptotic behavior of energy integrals under small perturbations of the boundary near corner points and conical points. Trudy Moskov. Mat. Obshch., 50, 1987, 79-129. (English transl.: Trans. Mosc. Math. Soc., 50, 1988, 77-127).

[28] Mazja W.G., Nazarov S.A., Plamenewski B.A. Asymptotische Theorie elliptischer Randwertaufgaben in singulär gestörten Gebieten. Bd. 1. Berlin: Akademie-Verlag. 1991. English translation: Asymptotic theory of elliptic boundary value problems in singularly perturbed domains. Vol.1, Birkhäuser Verlag, Basel, 2000.

[29] Mazja V.G., Plamenevskit B.A. On coefficients in asymptotics of solutions of elliptic boundary value problems in a domain with conical points. Math. Nachr., 76, 1977, 29-60. (Engl. transl. in Amer. Math. Soc. Transl. 123, 1984, 57-89).

[30] Mazja V.G., Plamenevski B.A. Estimates in $L_{p}$ and Hölder classes and the Miranda-Agmon maximum principle for solutions of elliptic boundary value problems in domains with singular points on the boundary. Math. Nachr. [in Russian], 81, 1978, 25-82. (English translasion in: Amer. Math. Soc. Transl. 123, 1984, 1-56).

[31] Milton, G. W. The theory of composites. Cambridge Monographs on Applied and Computational Mathematics, 6, Cambridge University Press, Cambridge, 2002, 719.

[32] Movchan A. B., Movchan N. V. Mathematical modelling of solids with nonregular boundaries. CRC Mathematical Modelling Series. CRC Press, Boca Raton, FL, 1995.

[33] Nazarov S.A. Asymptotic theory of thin plates and rods. Dimension reduction and integral estimates. Nauchnaya Kniga, Novosibirsk, 2001. 
[34] Nazarov S.A. Self-adjoint elliptic boundary-value problems. The polynomial property and formally positive operators. Probl. Mat. Anal., 16. St.-Petersburg: St.-Petersburg Univ., 1997, 167-192. (English transl.: J. Math. Sci. 92, (6) 1998, 4338-4353).

[35] Nazarov S.A. The polynomial property of self-adjoint elliptic boundary-value problems and the algebraic description of their attributes. Uspehi mat. nauk., 54, (5) 1999, 77-142. (English transl.: Russ. Math. Surveys., 54, (5) 1999, 947-1014)

[36] Nazarov S.A. Non-self-adjoint elliptic problems with the polynomial property in domains having cylindric outlets to infinity. Zap. Nauchn. Sem. St.-Petersburg Otdel. Mat. Inst. Steklov, 249, 1997, 212-231. (English transl.: J. Math. Sci., 101, (5) 1999, 35125-3522).

[37] Nazarov S.A. Asymptotic conditions at a point, self-adjoint extensions of operators and the method of matched asymptotic expansions. Trudy St.-Petersburg Mat. Obshch., 5, 1996, 112-183. (English transl.: Trans. Am. Math. Soc. 193, 1999, 77-126).

[38] Nazarov S.A. The damage tensor and measures. 1. Asymptotic analysis of anisotropic media with defects. Mekhanika tverd. tela., 3, 2000, 113-124. (English transl.: Mechanics of Solids, 35, (3) 2000, 96-105.)

[39] Nazarov S.A., Plamenevsky B.A. Elliptic problems in domains with piecewise smooth boundaries. Moscow: Nauka. 1991. (English transl.: Elliptic problems in domains with piecewise smooth boundaries. Berlin, New York: Walter de Gruyter. 1994).

[40] Nazarov S.A., SokoŁowski J. Asymptotic analysis of shape functionals J. Math. Pures Appl., $82,(2)$ 2003, 125-196.

[41] Nazarov S.A., Soko€owski J. Self-adjoint extensions for the Neumann Laplacian and applications. Acta Mathematica Sinica, 22, (3) 2006, 879-906.

[42] Nazarov S.A., SokoŁowski J. Spectral problems in the shape optimisation. Singular boundary perturbations. Asymptotic Analysis, 56(2008), No 3-4, 159-204.

[43] Nazarov S.A., SokoŁowski J. Spectral problems in elasticity. Singular boundary perturbations., submitted.

[44] Nazarov S.A., Sokołowski J., Specovius-Neugebauer M. General properties and shape sensitivity analysis of polarization matrices in elasticity. in preparation

[45] Norato J.A., Bendsøe M.P., Haber R.B., Tortorelli D.A. A topological derivative method for topology optimization. Struct Multidisc Optim 33, 2007, 375-386.

[46] Parton V.Z., Kudriavtsev B.A. Electromagnetoelasticity: Piezoelectrics and Electrically Conductive Solids. Gordon \& Breach Science Publishers Ltd, New York, 1998.

[47] PAZY A. Asymptotic expansions of ordinary differential equations in Hilbert space. Arch. Rational Mech. Anal. 24, 1967, 193-218.

[48] Polya G., Szegö G. Isoperimetric inequalties in mathematical physics. Annals of Mathematics Studies, 27, Princeton University Press, Princeton, N.J., 1951.

[49] SokoŁowski J., Zolesio J.P., Introduction to Shape Optimization. Shape Sensitivity Analysis., Springer Verlag, 1992.

[50] SokoŁowski J., Żochowski A. On topological derivative in shape optimization. SIAM Journal on Control and Optimization. 37, (4) 1999, 1251-1272. 
[51] SokoŁowski J., ŻochowskiA. Topological derivatives of shape functionals for elasticity systems. Mechanics of Structures and Machines. 29:333-351, 2001.

[52] Suo Z., Kuo C.M., Barnett D.M., Willis J.R. Fracture mechanics for piezoelectric ceramics. J. Mech. Phys. Solids. 40, (4) 1992, 739-765.

[53] Zorin I.S., Movchan A.B., Nazarov S.A. Application of the elastic polarization tensor in the problems of the crack mechanics. Mekhanika tverd. tela., 6, 1988, 128-134. (Russian) 